\title{
A New Representation in PSO for Discretisation-Based Feature Selection
}

\author{
Binh Tran, Student Member, IEEE, Bing Xue, Member, IEEE, and Mengjie Zhang, Senior Member, IEEE
}

\begin{abstract}
In machine learning, discretisation and feature selection are important techniques for preprocessing data to improve the performance of an algorithm on high-dimensional data. Since many feature selection methods require discrete data, a common practice is to apply discretisation before feature selection. In addition, for the sake of efficiency, features are usually discretised individually (or univariate). This scheme works based on the assumption that each feature independently influences the task, which may not hold in cases where feature interactions exist. Therefore, univariate discretisation may degrade the performance of the feature selection stage since information showing feature interactions may be lost during the discretisation process. Initial results of our previous proposed method (EPSO) showed that combining discretisation and feature selection in a single stage using bare-bones particle swarm optimisation can lead to a better performance than applying them in two separate stages. In this study, we propose a new method called PPSO which employs a new representation that can reduce the search space of the problem and a new fitness function to better evaluate candidate solutions to guide the search. The results on ten highdimensional datasets show that PPSO select less than $5 \%$ of the number of features for all datasets. Compared with the twostage approach which uses BBPSO for feature selection on the discretised data, PPSO achieves significantly higher accuracy on seven datasets. In addition, PPSO obtains better (or similar) classification performance than EPSO on eight datasets with a smaller number of selected features on six datasets. Furthermore, PPSO also outperforms the three compared (traditional) methods and performs similar to one method on most datasets in terms of both generalisation ability and learning capacity.
\end{abstract}

Index Terms-Feature selection, discretisation, particle swarm optimisation, classification, high-dimensional data.

\section{INTRODUCTION}

More and more high-dimensional datasets with thousands to tens of thousands of features become common in many machine learning applications in different fields such as bioinformatics, genomics, image processing and text classification [1]. These datasets usually have a significant number of redundant features and irrelevant features that can be considered as noise, which negatively affects the performance of the learning algorithm. Therefore, feature selection (FS) is usually a critical preprocessing step to select only relevant features for many machine learning tasks such as clustering and classification [1]. In this paper, we focus on FS for classification. Although many studies have shown the effectiveness of applying FS on high-dimensional data [1], [2], it is still challenging due to the large search space and the existence of feature interactions.

Binh Tran, Bing Xue, and Mengjie Zhang are with the Evolutionary Computation Research Group at Victoria University of Wellington, PO Box 600, Wellington, New Zealand (E-mail: binh.tran@ecs.vuw.ac.nz).
In addition to FS, discretisation is also crucial in preprocessing high-dimensional data. First of all, many learning algorithms are applicable to or efficient on discrete data only. Therefore, discretisation is used to transform features with continuous values into discrete or nominal values. Furthermore, via discretisation, minor fluctuations or possible noise in the data can be ignored. In this way, discretisation helps learning algorithms improve their effectiveness and efficiency [4]. Last but not least, since discrete data is more compact than continuous data, it requires less memory, and thus improving efficiency of learning algorithms. Therefore, FS and discretisation are commonly used in order to improve not only classification performance, but also computation time and storage requirement [5], [6].

Although many discretisation methods have been proposed, the most commonly used discretisation methods are univariate. By discretising one feature at a time, these methods are quite efficient when feature interactions do not exist [7]. When this assumption does not hold, it is necessary to discretise multiple features simultaneously. However, the time complexity of multivariate discretisation will be much higher. Therefore, a more powerful search technique is required to enable multivariate discretisation especially on high-dimensional data.

Furthermore, in practice, univariate discretisation is usually applied before FS as a requirement of many FS methods. However, univariate discretisation can destroy information about feature interactions which may be important for FS stage. Therefore, better representation may be obtained by merging the two processes in a single stage.

Particle swarm optimisation (PSO) is a meta-heuristic algorithm proposed by Kennedy and Eberhart [8]. PSO imitates social behaviours found in birds flocking. Many FS methods have been proposed using different types of PSO algorithms such as continuous PSO [9], [10] and binary PSO [11], [12], [13]. Results of these methods have shown high potential of PSO in this field. However, PSO has not been applied to discretisation.

In a recent method (EPSO) [14], we proposed to use a derived type of PSO called "bare-bones" PSO (BBPSO) [15] to simultaneously discretise and select features because of the following reasons. In PSO for FS, the PSO representation is usually a $N$-dimension vector corresponding to $N$ features. Each value is of the range $[0,1]$. If it is greater than a predefined threshold, the corresponding feature is selected and vice versa, regardless of how much smaller or greater it is when compared to the threshold. Therefore, two different evolved vectors may result in the same feature subset. On the other hand, in discretisation, a slightly different evolved cut-point 
may lead to a different discrete feature. As a result, finding a good cut-point requires a fine-tuned search mechanism which can be found in BBPSO. In this derived version of PSO, new positions are sampled using a Gaussian random generator with the center being the mean position of the individual best position (pbest) and its neighbours' best position (gbest) and standard deviation being the distance between them.

EPSO used BBPSO in a straightforward way to achieve discretisation and FS. One cut-point is evolved for each feature. Since a cut-point can be any value within the feature's range, the number of possible solutions in discretisation is much larger than FS. Therefore, entropy-based cut-points inspired from [16] are used as initial or potential cut-points to narrow the search space. The proposed method has shown promising results. However, with the proposed representation, the search space is still too big for BBPSO to obtain better performance. To narrow the search space, in this study, we propose a new method called PPSO which uses BBPSO to choose an appropriate cut-point for each feature among the potentially good ones. A new fitness function and a scaling mechanism are also proposed to improve the performance of the method.

\section{A. Goals}

This paper aims to develop a new method combining discretisation and feature selection in a single stage using BBPSO for high-dimensional continuous data. A new representation for particles in BBPSO is proposed to achieve this goal. While EPSO [14] used BBPSO to directly evolve a cut-point for each feature, the new approach called PPSO allows BBPSO to automatically choose potentially good cut-points for discretisation and FS. Performance of PPSO is compared with using full feature set, the two-stage approach of discretisation and FS, and EPSO on high-dimensional data. Our specific research objectives include:

1) How to perform multivariate discretisation and selection in a single stage to improve the feature set's discriminating power;

2) Whether the features discretised and selected by PPSO can obtain better classification accuracy than using full feature set;

3) Whether PPSO performs better than the corresponding two-stage approach in terms of accuracy, feature subset size and computation time;

4) Whether PPSO is more effective and efficient than EPSO;

5) Whether PPSO achieves better classification performance than traditional methods in both cases with and without FS bias ${ }^{1}$; and

6) Whether PPSO results are generalised well to the learning algorithms other than the wrapped method.

Although both the new method (PPSO) proposed in this work and the existing method (EPSO) in the conference paper [14] aim to tackle FS via discretisation, their major components, which are the representation and the evaluation

\footnotetext{
${ }^{1}$ FS bias is an important issue happened in FS when the whole set of data is used during the FS process; therefore, no unseen data is used to test the performance of the FS method [17].
}

method, are very different. In EC techniques, representation plays an important role in the effectiveness and efficiency of the method. A good representation can reduce the size of the search space, which is usually very large in EC approaches. The search space of FS problem alone is already very large, so combining it with discretisation results in an even larger space. Therefore, in this study, we propose a new representation so that the search process can be more effective. Another contribution of this work is a new fitness function that combines wrapper and filter measures in order to synthesise the strength of both approaches. The proposed hybrid approach is designed to better evaluate the candidate solution, however, without paying more computational cost by using wrapper and filter methods that are based on the same distance measure.

\section{BACKGROUND AND RELATED WORK}

This section provides background on FS and discretisation, introduces the entropy-based cut-points and the minimum description length principle which is employed in this study. It then presents methods that are closely related to our proposed method, FS via discretisation.

\section{A. Feature Selection}

Feature selection is essentially a combinatorial optimisation problem since there are $2^{N}$ possible different feature subsets to choose from a dataset with $N$ features. A FS method usually has two main components which are a search technique and a feature evaluation method.

In terms of feature evaluation, FS methods can be generally categorised into filter and wrapper approaches [18]. Filter approaches evaluate features based on their intrinsic characteristics. Examples of filter measures are distance [19], information gain [20], consistency [21], and correlation [22]. On the other hand, wrapper approaches use a learning algorithm to measure the classification performance of the selected features. Different learning algorithms can be used in this process, such as K-Nearest Neighbour [23], Decision Tree [24], and Support Vector Machines [25]. In general, filter methods are faster than wrapper methods; however, they usually obtain lower classification accuracy than wrappers [26].

Search techniques in FS methods can be divided into exhaustive search, heuristic search, random or stochastic search [27]. While exhaustive search guarantees to find the best solutions, it is infeasible for most real-world applications due to its high computation time. Sequential search such as Linear forward selection (LFS) [28] and greedy stepwise backward selection (GSBS) [29] are typical methods of heuristic search. These two methods are derived versions of sequential forward selection (SFS) [30] and sequential backward selection (SBS) [31], respectively. LFS [28] improved SFS effectiveness and efficiency by limiting the number of features considered in each step. Although backward selection can consider feature interactions better than forward selection [32], it is impractical to apply to datasets with a large number of features. GSBS cannot finish within a week when running on datasets with hundreds of features [33]. In additions, both forward and 
backward strategies usually face the problem of local optima [18].

Random search may generate subsets in a perfectly random fashion using Las Vegas algorithm such as LVW [34] which is too slow to converge in a large search space. Instead of randomly generation, evolutionary computation (EC) is a stochastic approach that applies evolutionary principle or swarm intelligence to generate better subsets from the current ones. Particle swarm optimisation (PSO) is a swarm intelligence technique that has been applied and shown their effectiveness in FS [35], [20]. Readers are referred to more comprehensive survey on different strategies for FS using EC techniques [36], [37]. Although PSO has been successfully applied to different optimisation problems including FS, it has not been applied to discretisation.

\section{B. Feature Discretisation}

Feature discretisation is a topic with a long research history. Many discretisation methods with different strategies have been proposed in this area. However, all of them share the same purpose which is to determine cut-points to partition features values into discrete values. Cut-points or split-points are real values within the range of the feature's values that are used to partition that range into several intervals. Existing discretisation methods can be categorised using different criteria [38], [39], [40]. In direct methods, intervals are generated based on a predefined parameter. On the other hand, incremental methods recursively split (or merge) intervals until some criteria is met creating splitting (or merging) approach. They are also known as top-down or bottom-up approaches, respectively. A discretisation method is supervised or unsupervised depending on whether class labels are used in the discretisation process or not. It is said to be global if the entire instance space is used in each discretisation step, or local if each discretisation step just uses a subset of instances. A method is also belong to univariate or multivariate depending on whether features are discretised individually or multiple features are discretised at the same time taking into account interactions between features [38], [40].

Equal-width and equal-frequency binning are two simple unsupervised methods. They discretise features into a predefined number of $m$ intervals with the same width or the same number of values, respectively. These simple methods are easy to implement but sensitive to value of $m$ which is usually difficult to determine especially when features are not uniformly-distributed or contain outliers [41].

Using the class labels as a guide in searching cut-points, supervised discretisation usually performs better than the unsupervised counterpart. 1R [42] defines cut-points are feature values lying at the boundary of different classes. Each bin needs to have at least six instances except for the last bin. In addition to different searching techniques, different evaluation measures are proposed such as classification error rates [46], [47], information gain [16], [43], and statistical measures [44], [45]. More comprehensive reviews can be found in [38], [39], [40], [48].

Among supervised methods, minimum description length (MDL) proposed by Fayyad and Irani [16] is one of the most popular used methods. It is an entropy-based incremental splitting discretisation method. Information gain is used to evaluate candidate cut-points. MDL recursively chooses the best cut-point to split one interval into two until the minimum description length principle (MDLP) is met. Inspired by this strategy, we propose to use the entropy-based cut-points that are accepted by MDLP as initial or candidate cut-points for BBPSO to evolve from.

\section{Entropy-based Cut Points}

Entropy-based discretisation aims to find the best splits so that the discretised features are as pure as possible in terms of class labels. This means that the majority of the values in one interval are preferred to have the same class label. If entropy $E(S)$ is used to measure the purity level of a set $S$, then according to this criterion, the cut-point with the highest information gain is the best one. Eq. (1) is used to calculate information gain of a cut-point $T$ for feature $A$ given $S$ as the set of feature $A$ values. $S_{1}$ and $S_{2}$ are subsets of $S$ after partitions.

$$
\operatorname{Gain}(T, A ; S)=E(S)-\frac{\left|S_{1}\right|}{|S|} E\left(S_{1}\right)-\frac{\left|S_{2}\right|}{|S|} E\left(S_{2}\right)
$$

Furthermore, not all cut-points are useful especially when the features are noisy or irrelevant to the class label. Therefore, Fayyad and Irani [16] proposed to use the MDLP as a criterion to accept a cut-point. According to MDLP, as shown in Eq. (2), a cut-point $T$ is only accepted if its information gain is greater than the cost of encoding the cut-point $T$ and the classes of the instances in the intervals induced by $T$. If the cut-point $T$ induces impure intervals, the sum of these two costs may exceed its information gain. As shown in Eq. (3), the latter cost $\delta(T, A ; S)$ will become larger if the entropy values $E\left(S_{1}\right)$ and $E\left(S_{2}\right)$ are larger. In noisy or irrelevant features, the cut-points usually can not induce intervals that are pure enough to satisfy the condition in Eq. (2). Therefore, using MDLP can deal with noise or irrelevant data.

$$
\operatorname{Gain}(T, A ; S)>\frac{\log _{2}(|S|-1)}{|S|}+\frac{\delta(T, A ; S)}{|S|}
$$

where

$\delta(T, A ; S)=\log _{2}\left(3^{k_{S}}-2\right)-\left[k_{S} E(S)-k_{S_{1}} E\left(S_{1}\right)-k_{S_{2}} E\left(S_{2}\right)\right]$

given $|S|$ as size of set $S, E(S)$ as the entropy of $S$, and $k_{S}$ as the number of classes appeared in $S$.

\section{Feature Selection via Discretisation}

Although FS and discretisation are emerging fields in recent decades, approaches that combine these tasks have not gained much attention. Chi2 [49] is one of the first methods proposing feature selection via discretisation. It is a bottom up method starting from intervals with only one feature value. Then adjacent intervals with the lowest $\chi^{2}$ test result will be merged recursively until $\chi^{2}$ values of all pairs exceeds a threshold. This threshold is determined by attempting to maintain a predefined consistency level of the data. By loosing this consistency level, Chi2 can come up with features that have only one interval, which can be removed for FS. Results on 
two synthetic datasets showed that Chi2 effectively discretised relevant features and removed all noise features. However, the user-defined inconsistency rate is hard to predefined and may also cause inaccuracy to the discretisation process [50]. Modified Chi2 [50] is a completely automatic discretisation method that address the drawbacks of Chi2.

Another approach of FS via discretisation is first ranking features based on some measures calculated during the discretisation process. Then, a number of top ranked features will be selected. An example of this approach is PEAR [51] in which features are ranked from those with the smallest number of cut-points to the largest. The top ranked features are considered as relevant and selected to form the final subset. Results showed that it has similar performance as the original feature set and better than Relief. However, it is difficult to choose appropriate parameters for PEAR and the number of features that should be selected to form the final subset. Similarly, in [52], features are ranked based on the ratio of the variance of the original continuous values and the number of bits used to encode the discrete feature.

In summary, feature selection via discretisation has been proposed in two separate stages. However, a single stage integrating them together has not been investigated yet.

\section{E. Particle Swarm Optimisation}

1) Continuous PSO: PSO proposed by Kennedy and Eberhart [8] is a population-based algorithm which maintains a swarm of particles. Each particle's position represents a complete candidate solution of the problem. It is actually a vector of $N$ real numbers, where $N$ is the dimensionality of the problem. In addition to position, each particle also has a velocity vector indicating its speed and direction that it should move in each dimension. These two elements are updated using Eqs. (4) and (5). As can be seen from these equations that particles are moving towards pbest and gbest.

$$
\begin{gathered}
v_{i d}^{t+1}=w * v_{i d}^{t}+c_{1} * r_{1 i}^{t} *\left(p_{i d}^{t}-x_{i d}^{t}\right)+c_{2} * r_{2 i}^{t} *\left(p_{g d}^{t}-x_{i d}^{t}\right) \\
x_{i d}^{t+1}=x_{i d}^{t}+v_{i d}^{t+1}
\end{gathered}
$$

where $v_{i d}^{t}$ and $x_{i d}^{t}$ are velocity and position of particle $i$ in dimension $d$ at time $t$, respectively. $p_{i d}^{t}$ and $p_{g d}^{t}$ are pbest and gbest positions in dimension $d$ at time $t . r_{1 i}^{t}$ and $r_{2 i}^{t}$ are uniformly distributed random values generated at time $t . c_{1}$ and $c_{2}$ are acceleration constants which determines the type of trajectory the particle travels, thus they are important to control the searching behaviour of the particle [53]. The inertia weight $w$ is used to control the impact of the last velocity to the current velocity.

2) Bare-bone PSO: As can be seen in Eq. (4) and (5) that PSO operates by sampling points in the search space. It uses pbest and gbest which are the discovered knowledge to guide the sampling. In [15], Kennedy investigated the trajectory of a single particle in a standard PSO where pbest and gbest were set as constants. All of its visited positions after a million iterations were plotted. The obtained histogram is a tidy bell curve centered midway between these constant pbest and gbest positions. The result suggests that trajectory of a particle is determined by the difference between its pbest and gbest. Therefore, the step size of a particle's movement should be based on these best positions. This finding resulted in a new PSO method called bare-bones PSO (BBPSO). In this method, particle's position is sampled using a Gaussian distribution $\mathcal{N}(\mu, \sigma)$ with the mean $\mu$ and standard deviation $\sigma$ as shown in Eq. (6).

$$
x_{i d}^{t+1}= \begin{cases}\mathcal{N}(\mu, \sigma), & \operatorname{rand}()<0.5 \\ p_{i d}^{t}, & \text { otherwise }\end{cases}
$$

where $\mu$ is the center of pbest and gbest and $\sigma$ is the absolute difference between these best positions. The $\operatorname{rand}()$ function is used to speed up convergence by retaining the previous best position pbest.

By using the Gaussian number generator, BBPSO avoids the need to optimise the velocity vector and the delay of position adaptation. Although PSO and BBPSO have been proposed for FS ([54], [37], [55]), both have not been proposed for discretisation or discretisation and FS simultaneously.

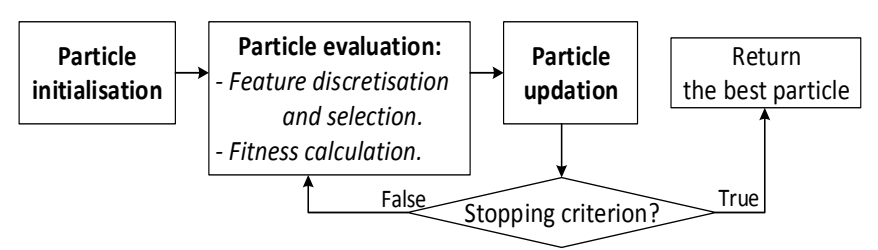

Fig. 1. EPSO and PPSO basic steps.

\section{The Proposed APPROACH}

This section describes EPSO and PPSO. Both methods follow the basic steps shown in Fig. 1. After initialisation, particles are iteratively evaluated and updated until the stopping criteria is met. In order to evaluate a particle, the training data is first discretised and features are selected based on the evolved cut-points. The transformed data is then put into a learning algorithm to calculate the fitness. Based on this fitness, pbest and gbest are updated and used to update a particle's position as described in Eq. (6).

Discretisation and FS steps in both methods work in the same principle. To achieve discretisation, a feature value is converted/discretised into 0 if it is smaller than the evolved cut-point; otherwise, it will be 1 . If all values of a feature are converted into the same discrete value, it is considered as an irrelevant feature because it can not distinguish instances of different classes. Feature selection is done by eliminating these useless features. The remaining discrete features are evaluated based on the improvement in classification performance of the whole discretised data.

\section{A. The EPSO Method}

The main idea of EPSO is using BBPSO to directly evolve a cut-point which can be any real value falling in the range of the corresponding feature values $\left[\operatorname{Min}_{F} . . M a x_{F}\right]$. Position of each particle represents a candidate solution which is a real vector with $N$-dimension corresponding to the dimensionality of the problem. Fig. 2 shows an example of a particle's 


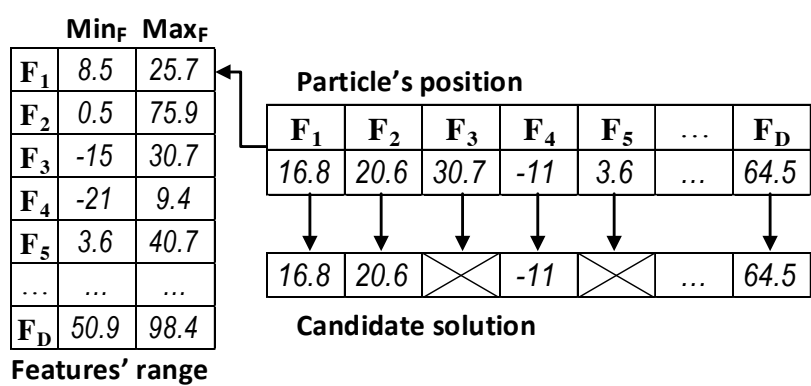

Fig. 2. Particle representation of EPSO.

position and its corresponding candidate solution. In this example, the first dimension of the particle, which represents the cut-point for the first feature $\left(F_{1}\right)$, needs to have a value within the range [8.5, 25.7]. If an updated cut-point of a feature $F$ is out of this range, it will be set to the nearest boundary.

1) Particle Initialisation: Because the search space of multivariate discretisation on high-dimensional data is huge, each initial particle is restricted to a random feature subset of size 50 for binary-class problems and size 150 for multiclass problems as suggested in [56]. This means that for those features that are not selected in the initial candidate solutions, their cut-points will be set to the maximum values of the corresponding features. For the other selected features, their cut-points are initialised using the best entropy-based cut-point that satisfies MDLP [16] (see Section II-C). In principle, they can be initialised based on any value within the range of the corresponding feature. However, completely random initial cut-points may lead to slow convergence. Furthermore, information gain of the best cut-point of a feature is an indicator of its relevancy. Therefore, features with larger information gain will have higher probability to be selected in the initialisation procedure.

2) Particle Evaluation: Based on the cut-points evolved by the particle, the training data is transformed into a new training set with discrete values and a smaller number of features thanks to eliminating features whose cut-points are equal to the minimum or maximum values. For example, in Fig. $2, F_{3}$ cut-point is equal to its maximum value and $F_{5}$ cut-point is equal to its minimum value, both features will be discarded.

The discretisation and feature selection solution in each particle is then evaluated based on the classification accuracy of the transformed training set. By evaluating the whole discrete data, the proposed method is able to evaluate cut-points of all the selected features as well as consider feature interactions at the same time. Fitness function uses the balanced classification accuracy [57] as follows:

$$
\text { balanced_accuracy }=\frac{1}{c} \sum_{i=1}^{c} \frac{T P_{i}}{\left|S_{i}\right|}
$$

where $c$ is the number of classes of the problem, $T P_{i}$ is the number of correctly identified instances in class $i$ and $\left|S_{i}\right|$ is the sample size of class $i$. All classes are treated equally with the weight of $1 / c$

\begin{tabular}{|c|c|c|c|c|c|c|c|c|c|c|c|}
\hline \multicolumn{6}{|c|}{ Potential cut-point table } & \multirow{2}{*}{\multicolumn{6}{|c|}{ Cut-point index }} \\
\hline & & & $\mathrm{C}_{2}$ & $\mathrm{C}_{3}$ & $\mathrm{C}_{4}$ & & & & & & \\
\hline$F_{1}$ & 2 & 5.25 & 6.8 & & & \multicolumn{6}{|c|}{ Particle's position } \\
\hline$F_{2}$ & 3 & 107 & 24.9 & 5 & & $\mathbf{F}_{1}$ & $\mathbf{F}_{2}$ & $\mathbf{F}_{3}$ & $\mathbf{F}_{4}$ & ... & $F_{D}$ \\
\hline$F_{2}$ & & 10.1 & & & & 2 & 0 & 3 & 0 & $\ldots$ & 2 \\
\hline$F_{3}$ & 4 & -0.45 & 0.67 & 5.2 & 20.5 & & & & & & \\
\hline $\mathbf{F}_{4}$ & 1 & -32.5 & & & & & & & & & \\
\hline$\ldots$ & & & & & & 6.8 & & 5.2 & $x$ & $\ldots$ & -1.54 \\
\hline$F_{D}$ & 3 & -3.72 & -1.54 & 6.55 & & & ate & & & & \\
\hline
\end{tabular}

Fig. 3. Particle representation of PPSO.

\section{B. The Proposed Method: PPSO}

In EPSO, BBPSO is free to evolve a cut-point within the range of the corresponding feature. This may result in a huge search space especially in a multivariate approach on highdimensional data. Therefore, to narrow the search space into highly potential areas, in PPSO, we use BBPSO to choose a cut-point from potential cut-points of each feature. Potential cut-points are entropy-based cut-points that have their information gain satisfying the MDLP criterion shown in Eq. (2). Each feature may have a different number of potential cutpoints which are calculated and stored in a potential cut-point table. Fig. 3 shows an example of this table and a particle's position as well as the corresponding candidate solution. Each particle position is an integer vector denoting the chosen cutpoint indexes. The size of vector, therefore, is equal to the number of the original features and the evolved value needs to be between 1 and the number of potential cut-points of the corresponding feature. For example, in Fig. 3, the first feature $\left(F_{1}\right)$ has two potential cut-points with index 1 and 2 . Therefore, the first dimension of the particle needs to fall in the range $[1,2]$. If it is 2 as in the example, then the cut-point 6.8 is chosen to discretise $F_{1}$.

During the updating process, if the updated value of a dimension is outside the cut-point index range, then it is set to 0 which indicates that the corresponding feature does not have a good cut-point and therefore should be ignored.

1) Particle Initialisation: Each particle position is initialised as a random feature subset with a restricted number of selected features which have their cut-point indexes different from 0 . The selected features will have their cut-point index set to the index of the best MDLP cut-point (see Section II-C) [16]. Similar to EPSO, features with higher information gain will have higher chance to be selected.

In order to make PPSO general to all problems, PPSO uses one restricted size for all datasets. Then during the evolutionary process, when BBPSO seems to get stuck in local optima, BBPSO will be reset with a larger size if the current gbest fitness is better than the last gbest fitness at least $10 \%$. The aim of this scaling mechanism is to start searching from small feature subsets while opening chances for larger and better feature subsets.

2) Particle Evaluation: Based on the chosen cut-point index for each feature, the cut-point value is retrieved from the potential cut-point table. It is then used to discretise the 
corresponding feature. However, if the evolved cut-point index of a feature is 0 , that feature is considered as not selected. Therefore, in Fig. 3, Features $F_{2}$ and $F_{4}$ are not selected in this example.

In EPSO, classification accuracy is used as the fitness measure to evaluate each particle. This may be difficult to distinguish cases where the boundary between classes is quite large, enabling many different models to obtain the same accuracy. Furthermore, while the wrapper methods can produce solutions with high accuracy, filter methods are usually faster and general. Combining the strength of these two approaches in the evaluation function is expected to produce better solutions. In addition, combining the two measures can also better distinguish the subtle difference between feature subsets, providing a smoother fitness landscape to facilitate the search process. However, simply combining these measures may be impractical in terms of computation. Therefore, we need to find a smart way to combine them without requiring more running time. Among the commonly used filter measures, distance is a multivariate measure that can evaluate the discriminating ability of a feature set and it is used as the base measure of KNN. Therefore, incorporating this measure with the KNN wrapper method will not increase the computation time because the distance measure is calculated only once but used twice.

In this study, the proposed fitness function uses both balanced classification accuracy (Eq. 7) and a distance measure with a weighting coefficient $(\mu)$ as shown in Eq. (8). The distance measure [58], as shown in Eq. (9), is used to maximise the distance between instances of different classes $\left(D_{B}\right)$ and minimise the distance between instances of the same class $\left(D_{W}\right) . D_{B}$ and $D_{W}$ are calculated by using Eq. (10) and (11), respectively.

$$
\begin{gathered}
\text { fitness }=(\mu * \text { balanced_accuracy }+(1-\mu) * \text { distance }) \\
\text { distance }=\frac{1}{1+\exp ^{-5\left(D_{B}-D_{W}\right)}} \\
D_{B}=\frac{1}{|S|} \sum_{i=1}^{|S|}\left\{\min _{\left\{j \mid j \neq i, \operatorname{class}\left(V_{i}\right) \neq \operatorname{class}\left(V_{j}\right)\right\}} \operatorname{Dis}\left(V_{i}, V_{j}\right)\right. \\
D_{W}=\frac{1}{|S|} \sum_{i=1}^{|S|} \max _{\left\{j \mid j \neq i, \operatorname{class}\left(V_{i}\right)=\operatorname{class}\left(V_{j}\right)\right\}} \operatorname{Dis}\left(V_{i}, V_{j}\right)
\end{gathered}
$$

where $\operatorname{Dis}\left(V_{i}, V_{j}\right)$ is the distance between two vectors $V_{i}$ and $V_{j}$. In this study we use the proportion of matches or overlaps between two binary vectors as the distance between them.

As can be seen in Eq. (10) and (11), $D_{B}$ and $D_{W}$ are the average distance between each instance to its nearest miss and to its farthest hit, respectively. While both of them are in the range [0,1], 1 is the best case of $D_{B}$ and the worst case for $D_{W}$. Therefore, Eq. (9) maximises $D_{B}$ and minimises $D_{W}$ to find feature subsets showing a close distance between instances of the same class and a far distance between instances of different classes.

The logistic function with coefficient -5 as shown in the right plot of Fig. 4 is used to transform the difference between $D_{B}$ and $D_{W}$ in the range $[-1,1]$ into a value in the full range
$[0,1]$ in which 0 is the worst distance and 1 is the best distance. Note that the logistic function with coefficient -1 shown in the left plot of Fig. 4 does not return a value in the full range $[0,1]$ for an input between -1 and 1 .

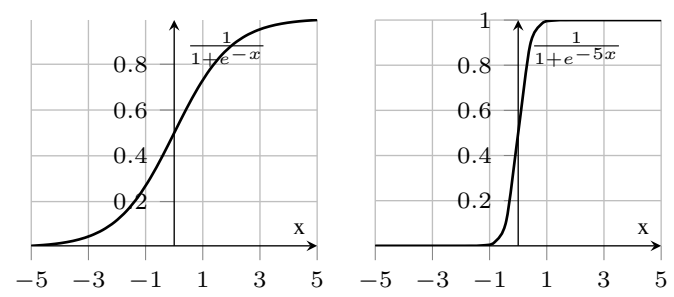

Fig. 4. Logistic function $f(x)=\frac{1}{1+e^{-x}}, f(x)=\frac{1}{1+e^{-5 x}}$

\section{The Overall Approach}

An overview of the proposed approach to discretisation and FS in one stage is shown in Fig. 5(a). The two-stage approach (named PSO-FS) is shown in Fig. 5(b) in which features are first discretised and then selected. Except for the details inside the dotted box, both systems have the same structure. Firstly, the dataset is split into a training and a test set. Based on the training set, the evolved cut-points for the selected features are returned and used to transform the training and the test sets. These transformed datasets are input to the classification algorithms for performance evaluation of both methods. Algorithm 1 and Algorithm 2 present the pseudo-code of EPSO and PPSO, respectively.

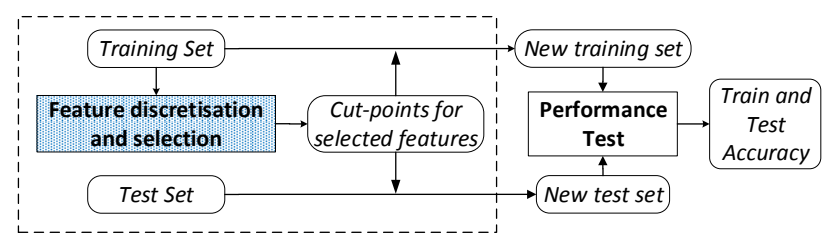

(a) EPSO and PPSO (Discretisation and FS in a single stage).

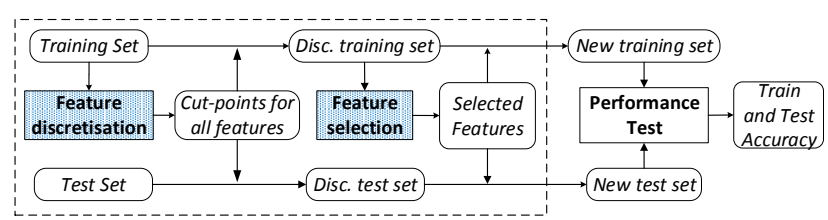

(b) PSO-FS (Discretisation and FS in two stages).

Fig. 5. System Overview.

Both EPSO and PPSO follow the overall structure described in Fig. 5(a), but are very different in the key aspects. The major differences are as follows. First of all, the representation scheme of PPSO significantly reduces the search space of the problem when considering only the potentially good cutpoints. It improves not only the effectiveness but also the efficiency. Secondly, the fitness function of PPSO is enforced with a distance measure to provide better evaluation. Thirdly, the initialisation procedure in PPSO is more general than EPSO when using one restricted size for all problems to initialise 

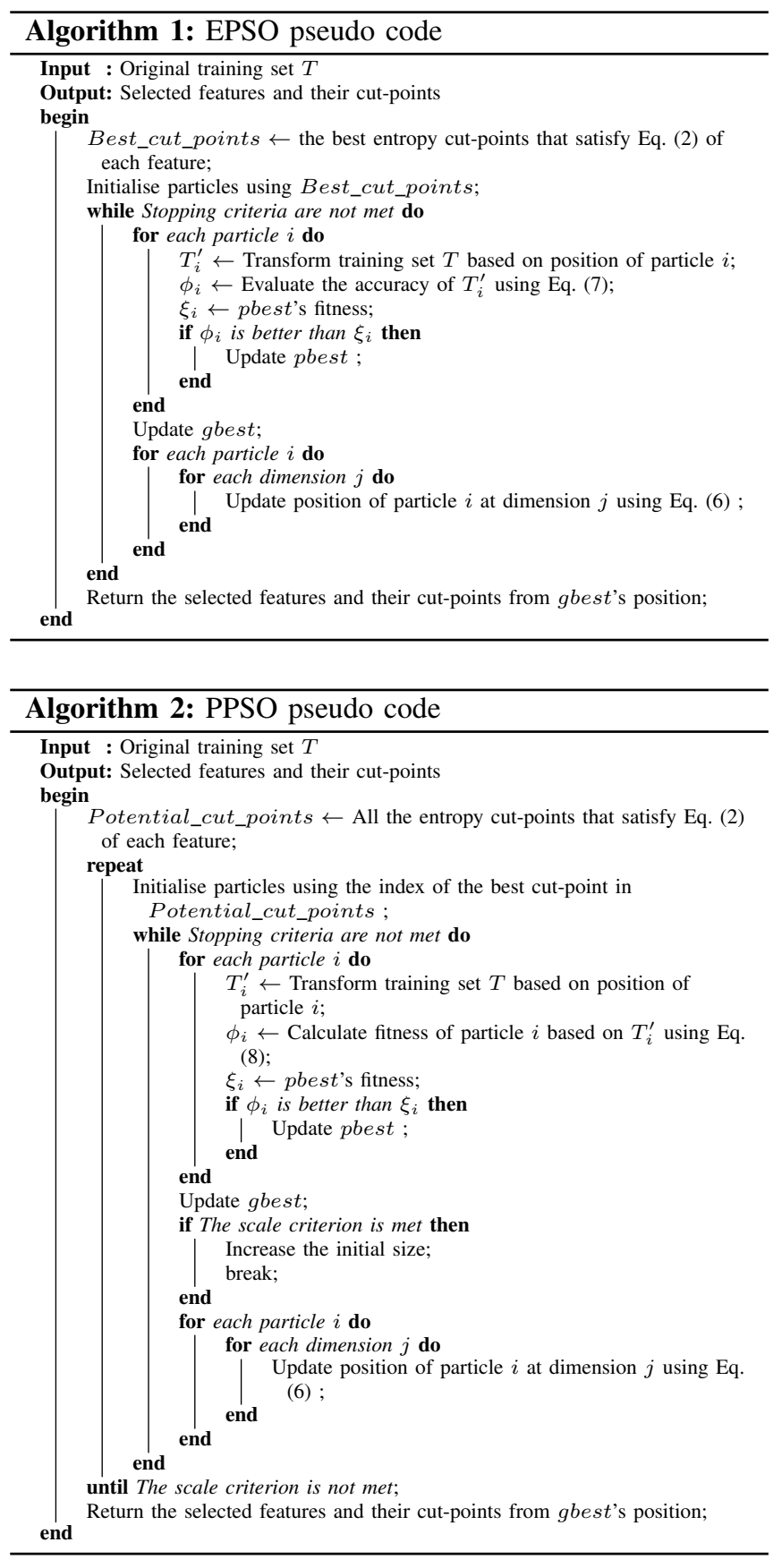

particles. Finally, PPSO uses scaling mechanism to move the search focus to larger feature subsets when needed. These new ideas in PPSO are expected to improve its performance in terms of the classification accuracy, the number of selected features and the computation cost.

\section{EXPERIMENT DESIGN}

This section describes the details related to the experiments including the datasets, the baseline methods used to compare with our methods, the parameter settings, the termination criteria as well as the configuration of the experiments.

1) Datasets: To test PPSO performance on highdimensional data, we used ten gene expression datasets which
TABLE I

DATASETS

\begin{tabular}{|l|r|r|r|r|r|}
\hline Dataset & \#Features & \#Instances & \#Classes & $\begin{array}{c}\text { \%Smallest } \\
\text { class }\end{array}$ & $\begin{array}{c}\text { \%Largest } \\
\text { class }\end{array}$ \\
\hline SRBCT & 2,308 & 83 & 4 & 13 & 35 \\
\hline DLBCL & 5,469 & 77 & 2 & 25 & 75 \\
\hline 9Tumor & 5,726 & 60 & 9 & 3 & 15 \\
\hline Leukemia 1 & 5,327 & 72 & 3 & 13 & 53 \\
\hline Brain Tumor 1 & 5,920 & 90 & 5 & 4 & 67 \\
\hline Leukemia 2 & 11,225 & 72 & 3 & 28 & 39 \\
\hline Brain Tumor 2 & 10,367 & 50 & 4 & 14 & 30 \\
\hline Prostate & 10,509 & 102 & 2 & 49 & 51 \\
\hline Lung Cancer & 12,600 & 203 & 5 & 3 & 68 \\
\hline 11Tumor & 12,533 & 174 & 11 & 4 & 16 \\
\hline
\end{tabular}

TABLE II

PSO PARAMETER SETTINGS

\begin{tabular}{|l|l|}
\hline Parameters & Settings \\
\hline Population Size & \#features/20 (restriction to 300) \\
Maximum iterations & 70 \\
$\begin{array}{l}\text { Stopping criterion } \\
\text { Communication topology }\end{array}$ & gbest not improved for 10 iterations \\
Scaling criterion (PPSO only) & $\begin{array}{l}\text { Fully connected } \\
\text { gbest not improved for 10 iterations and the } \\
\text { current gbest fitness is better than the previous } \\
\text { gbest fitness at least 1\% }\end{array}$ \\
\hline
\end{tabular}

are available on http://www.gems-system.org. Table I describes details about these datasets.

2) Baseline Methods: To test the effectiveness of PPSO in discretisation and FS, we compared the classification performance of $\mathrm{KNN}$ on the transformed dataset by PPSO, the original dataset, and the transformed dataset by EPSO. We also compared PPSO with the two-stage method (PSO-FS) to see whether the single stage approach has better performance than the two-stage one. In PSO-FS, MDL [16] is used to discretise data before applying PSO for feature subset selection. PPSO is also compared with several traditional two-stage methods which combines MDL [16] for discretisation with LFS [28], with Consistency method [21] and Correlation based feature selection method (CFS) [22] for FS. We also compared PPSO with the Modified Chi2 (or MChi2) [50] which is a representative method for FS via discretisation. We used the Weka package [29] to run LFS, CFS and Consistency, and the KEEL package [59] to run MChi2.

3) Parameter Settings and Termination Criteria: Parameter settings for the three compared methods, PSO-FS, EPSO and PPSO, are described in Table II. Since the size of the search space is proportional to the dimensionality of the problem which varies from one dataset to another. The number of features in the ten datasets ranges from about 2,000 to 12,000, leading to very different sizes of search space. Therefore, we set the population size as the number of features divided by 20 with a restriction to 300 due to the limited computer memory. The maximum number of iterations was set to 70; however, an early stop was also applied when gbest is not improved after 10 iterations. The scaling criterion in PPSO is when gbest is not improved after 10 iterations and the current gbest fitness is better than the previous gbest fitness for at least $1 \%$. PPSO starts with the initial size of 150 which is suggested in [56] for multi-class datasets. However, based on our experiments, this value is also a good initial size for binary class problems since PSO is able to choose an appropriate feature subset size during the evolutionary process. This initial size increases 50 every time the scaling criterion is met. 
4) Experiment Configuration: As a wrapper approach, PPSO can use the classification performance of any learning algorithm to evaluate particles. In this work, k-nearest neighbour (KNN) with $\mathrm{k}=1$ was used because it is simple, fast, and non-parametric.

Since these datasets have small sample sizes, we used 10fold cross validation $(\mathrm{CV})$ to generate training and test set. In each cross validation, one fold is used to form test set and the remaining nine folds are used to form training set. Test set is used for performance evaluation of the discretisation and FS solution produced by each method based on the training set. During the evolutionary process, an inner loop of 10-fold CV on the training set is used for fitness evaluation. Therefore, each method comprises of two loops of $\mathrm{CV}$ as recommended in [18] to avoid FS bias.

To eliminate statistical variations, each method was run 30 times with different random seeds for each dataset. Because each dataset was split into training and test set using 10 fold $\mathrm{CV}$, a total of 300 runs were executed for each method. Experiments were run on PC with Intel Core i7-4770 CPU @ $3.4 \mathrm{GHz}$ and $8 \mathrm{~GB}$ memory. The results of 30 runs from each method were compared using the statistical Wilcoxon significance test with $5 \%$ significance level.

TABLE III

AVERAGE TEST RESULTS.

\begin{tabular}{|c|c|c|c|c|c|}
\hline Dataset & Method & Size & Best & Mean (Std) & $S$ \\
\hline \multirow{4}{*}{ SRBCT } & Full & 2308.0 & & 87.08 & - \\
\hline & PSO-FS & 150.0 & 97.50 & $91.31(2.71)$ & - \\
\hline & EPSO & 137.3 & 100.00 & 96.89 (1.64) & + \\
\hline & PPSO & 108.5 & 100.00 & $95.78(1.96)$ & \\
\hline \multirow{4}{*}{ DLBCL } & Full & 5469.0 & & 83.00 & - \\
\hline & PSO-FS & 101.8 & 96.67 & 80.03 (6.13) & - \\
\hline & EPSO & 42.8 & 94.17 & $85.18(5.46)$ & $=$ \\
\hline & PPSO & 44.0 & 94.17 & $86.22(3.58)$ & \\
\hline \multirow{4}{*}{ 9Tumor } & Full & 5726.0 & & 36.67 & - \\
\hline & PSO-FS & 955.0 & 55.00 & 45.95 (4.93) & - \\
\hline & EPSO & 138.5 & 65.00 & $58.22(3.12)$ & $=$ \\
\hline & PPSO & 118.1 & 65.00 & $59.28(2.08)$ & \\
\hline \multirow{4}{*}{ Leuk1 } & Full & 5327.0 & & 79.72 & - \\
\hline & PSO-FS & 150.0 & 92.22 & $81.60(4.72)$ & - \\
\hline & EPSO & 135.9 & 95.56 & $93.37(1.83)$ & - \\
\hline & PPSO & 80.4 & 95.42 & $94.37(1.36)$ & \\
\hline \multirow{4}{*}{ Brain1 } & Full & 5920.0 & & 72.08 & - \\
\hline & PSO-FS & 317.3 & 78.75 & $71.00(3.06)$ & - \\
\hline & EPSO & 150.7 & 79.17 & 72.79 (3.48) & $=$ \\
\hline & PPSO & 73.4 & 82.08 & $74.40(3.67)$ & \\
\hline \multirow{4}{*}{ Leuk2 } & Full & 11225.0 & & 89.44 & - \\
\hline & PSO-FS & 150.0 & 93.89 & $86.11(3.97)$ & - \\
\hline & EPSO & 139.9 & 94.44 & $89.93(2.79)$ & - \\
\hline & PPSO & 86.7 & 100.00 & $96.74(1.64)$ & \\
\hline \multirow{4}{*}{ Brain2 } & Full & 10367.0 & & 62.50 & - \\
\hline & PSO-FS & 417.9 & 82.08 & $69.11(5.89)$ & $=$ \\
\hline & EPSO & 152.8 & 83.75 & 70.76 (5.30) & $=$ \\
\hline & PPSO & 66.7 & 74.58 & $68.75(4.24)$ & \\
\hline \multirow{4}{*}{ Prostate } & Full & 10509.0 & & 85.33 & - \\
\hline & PSO-FS & 777.4 & 90.33 & $85.20(2.35)$ & - \\
\hline & EPSO & 54.9 & 90.33 & $83.74(3.55)$ & - \\
\hline & PPSO & 65.6 & 95.17 & $91.82(1.77)$ & \\
\hline \multirow{4}{*}{ Lung } & Full & 12600.0 & & 78.05 & - \\
\hline & PSO-FS & 686.2 & 85.73 & $81.72(2.08)$ & + \\
\hline & EPSO & 150.8 & 85.58 & $80.60(2.42)$ & $=$ \\
\hline & PPSO & 203.0 & 84.11 & $79.38(3.26)$ & \\
\hline \multirow{4}{*}{ 11Tumor } & Full & 12533.0 & & 71.42 & \\
\hline & PSO-FS & 1638.8 & 86.07 & $82.62(1.70)$ & + \\
\hline & EPSO & 149.9 & 83.68 & 79.29 (2.11) & + \\
\hline & PPSO & 167.0 & 83.20 & $76.83(2.91)$ & \\
\hline
\end{tabular}

\section{RESUlTS AND DisCUSSIONS}

Table III shows the results of PSO-FS, EPSO, and PPSO. The average feature subset size returned by each method over the 30 runs is shown in column "Size". The best, mean and standard deviation of KNN accuracies using "Full" (i.e. all continuous features), or using the transformed data by each of the compared methods are shown in columns four and five. The reported results are test accuracies calculated based on Eq. (7). For each dataset, the best accuracy and the smallest size are highlighted in bold.

Column $S$ displays the statistical Wilcoxon significance test results of the method in the corresponding row over PPSO. "+" or "-" means the result is significantly better or worse than PPSO. "=" means they have similar performance. In other words, the more "-", the better the proposed approach. The same meaning of "+" and "-" will be used throughout this paper.

\section{A. PPSO versus Full}

As can be seen from Table III that the average numbers of features selected by PPSO are significantly smaller than the total number of features. PPSO selects less than $1 \%$ of the total number of features on four datasets, less than $2 \%$ on five datasets and $4.6 \%$ on SRBCT. In general, PPSO achieves the smallest subsets on six datasets

With the discretised and selected features, PPSO achieves significantly better classification performance than using all continuous features on all ten datasets. It increases more than $5 \%$ accuracy on seven out of ten datasets with the highest improvement of $23 \%$ on 9Tumor.

The results indicate that PPSO can produce a much more powerful and compact representation for high-dimensional datasets by simultaneously discretise and select relevant features in a single stage.

\section{B. PPSO versus $P S O-F S$}

In terms of dimensionality reduction, feature subsets returned by PPSO are much smaller than those selected by PSOFS which is the two-stage approach. In terms of classification accuracy, the PPSO transformed data outperforms PSO-FS on seven datasets. While PSO-FS degrades the classification performance on Brain1 and Prostate, PPSO still obtains better accuracies than using "Full" on these dataset with about $11 \%$ and $8 \%$ the number of features selected by PSO-FS on these datasets respectively. Similar pattern can be seen in DLBCL and Leuk2 datasets.

Results of 9Tumor dataset reveal the largest difference between two approaches. While the transformed dataset by PSOFS improves 9\% classification accuracy with 955 features, the transformed dataset by PPSO achieves $23 \%$ improvement using eight times less features than PSO-FS (118 features). A similar pattern is witnessed in the first six datasets.

In Brain2, PPSO obtains a similar accuracy to PSO-FS with a quarter of features selected by PSO-FS. In Lung and 11Tumor, PPSO obtains worse results than PSO-FS with about one third and one tenth number of features selected by PSOFS, respectively.

In general, solutions evolved by the proposed one-stage approach obtain either a significantly better or similar classification accuracy to the two-stage approach on eight out of the ten datasets. 


\section{PPSO versus EPSO}

As can be seen from Table III that PPSO selects a smaller number of features than EPSO on six datasets while obtaining better or similar classification accuracy to EPSO on eight datasets. In Leuk2, while EPSO obtains a similar classification accuracy to "Full" with 140 features, PPSO improves 7\% accuracy with only 87 features.

The improvement of PPSO over EPSO may be resulted from several proposed strategies in PPSO. First of all, PPSO only finds an appropriate cut-point for each feature from the potentially good cut-points while EPSO chooses any possible cutpoint within the range of the feature values. Therefore, PPSO has a smaller search space than EPSO. This enables PPSO to better cover the search space when using the same parameter setting as EPSO. Furthermore, the evolutionary process of PPSO is not only guided by accuracy of the transformed dataset but also the distance measure. This measure helps PPSO to differentiate feature subsets with the same training accuracy. Finally, the scaling mechanism enables PPSO to move the evolutionary process to larger feature subsets which may provide better solutions.

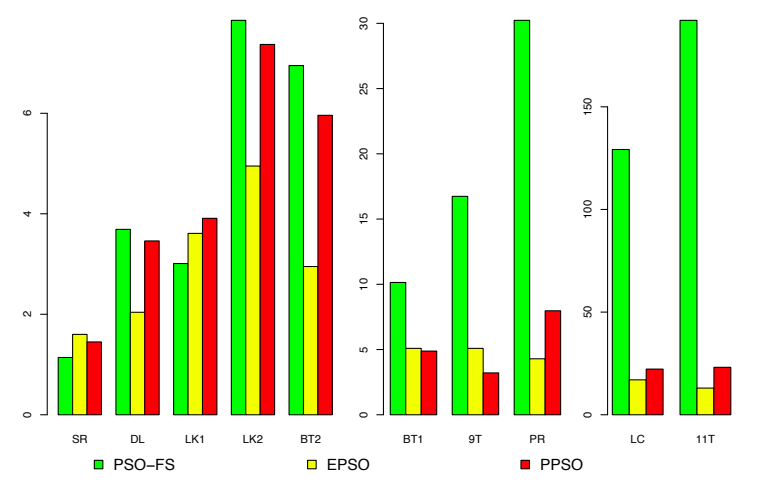

Fig. 6. Running Time of PPSO.

\section{Computation Time}

Fig. 6 compares the running time to complete a single run of PSO-FS, EPSO, and PPSO. The reported time (in minutes) is the average of 30 runs. Note that the three compared methods use the same wrapper approach with KNN for fitness evaluation. We also note that to evaluate each particle, PSO-FS only needs to transform data based on the selected features, while PPSO needs to discretise and select features at the same time. This means that PPSO evaluation time is slightly longer than PSO-FS. Therefore, PPSO is intended to run longer than PSO-FS. However, an opposite trend is reported in Fig. 6.

Compared with PSO-FS, the running time of PPSO is shorter on eight datasets with about 8 times shorter in 11Tumor dataset. As can be seen from Fig. 6 that the larger the dataset, the greater the ratio of PSO-FS's running time to PPSO's running time. An investigation of the evolutionary process reveals the main reason of this phenomenon. It is that the evolved feature subsets by PPSO are much smaller than those by PSO-FS, which in turn leads to a much smaller running time required by $\mathrm{KNN}$ in fitness evaluation process.
PPSO takes slightly longer time than EPSO on most datasets due to the scaling mechanism which introduces large feature subsets. It is noticed that although the fitness function of PPSO combines both wrapper and filter measures, the time spending for fitness evaluation is not longer than EPSO since both KNN and the filter measure are calculated based on the distance measure which is calculated once and used for both measures. Furthermore, since the number of features selected by PPSO is smaller, the test process will be faster as well.

TABLE IV

COMPARISON With TRADitional Methods.

\begin{tabular}{|c|c|c|c|c|c|c|c|c|c|}
\hline \multirow{2}{*}{ Dataset } & \multirow{2}{*}{ Method } & \multirow{2}{*}{ Size } & \multicolumn{3}{|c|}{ Training } & \multicolumn{3}{|c|}{ Test } & \multirow{2}{*}{ Time(s } \\
\hline & & & Best & Mean & $S_{T r}$ & Best & Mean & $S_{T e}$ & \\
\hline \multirow{6}{*}{ SRBCT } & Full & $2,308.0$ & & 83.35 & - & \multirow[b]{6}{*}{100.00} & 87.08 & - & \\
\hline & MDL+LFS & 6.1 & & 98.19 & - & & 88.75 & - & 28.78 \\
\hline & MDL+CON & 4.3 & & 97.71 & - & & 85.83 & - & 3.24 \\
\hline & MDL+CFS & 80.9 & & 100.00 & $=$ & & 100.00 & + & 318.04 \\
\hline & MChi2 & 85.9 & & 100.00 & $=$ & & 100.00 & + & 83.07 \\
\hline & PPSO & 108.5 & 100.00 & 100.00 & & & 95.78 & & 87.14 \\
\hline \multirow{6}{*}{ DLBCL } & Full & $5,469.0$ & & 81.71 & - & \multirow[b]{6}{*}{94.17} & 83.00 & - & \\
\hline & MDL+LFS & 4.0 & & 98.24 & - & & 74.00 & - & 73.78 \\
\hline & MDL+CON & 3.4 & & 98.14 & - & & 92.50 & + & 6.29 \\
\hline & MDL+CFS & 58.0 & & 99.22 & - & & 91.67 & + & 1310.49 \\
\hline & MChi2 & 10.2 & & 87.96 & - & & 75.50 & - & 369.45 \\
\hline & PPSO & 44.0 & 100.00 & 100.00 & & & 86.22 & & 207.40 \\
\hline \multirow{6}{*}{ 9Tumor } & Full & $5,726.0$ & & 33.44 & - & \multirow[b]{6}{*}{65.00} & 36.67 & - & \\
\hline & MDL+LFS & 12.6 & & 82.39 & - & & 41.67 & - & 64.20 \\
\hline & MDL+CON & 7.6 & & 71.61 & - & & 28.33 & - & 12.65 \\
\hline & MDL+CFS & 38.0 & & 90.71 & - & & 53.33 & - & 656.21 \\
\hline & MChi2 & 58.5 & & 77.28 & - & & 48.33 & - & 260.70 \\
\hline & PPSO & 118.1 & 92.87 & 92.31 & & & 59.28 & & 192.68 \\
\hline \multirow{6}{*}{ Leuk1 } & Full & $5,327.0$ & & 79.77 & - & & 79.72 & - & \\
\hline & MDL+LFS & 4.8 & & 99.17 & - & & 81.39 & - & 70.48 \\
\hline & MDL+CON & 3.0 & & 98.67 & - & & 89.17 & - & 5.69 \\
\hline & MDL+CFS & 56.0 & & 100.00 & $=$ & & 93.19 & - & 1358.35 \\
\hline & MChi2 & 46.4 & & 98.80 & - & & 92.08 & - & 276.35 \\
\hline & PPSO & 80.4 & 100.00 & 100.00 & & 95.42 & 94.37 & & 236.56 \\
\hline & Full & $5,920.0$ & & 65.07 & - & & 72.08 & - & \\
\hline & MDL+LFS & 9.9 & & 89.13 & - & & 59.17 & - & 104.41 \\
\hline Braml & MDL+CON & 6.2 & & 79.99 & - & & 55.42 & - & 14.51 \\
\hline & MDL+CFS & 115.4 & & 99.93 & - & & 79.58 & + & 2859.76 \\
\hline & MChi2 & 290.0 & & 80.63 & - & & 74.58 & $=$ & 438.82 \\
\hline & PPSO & 73.4 & 100.00 & 99.99 & & 82.08 & 74.40 & & 292.84 \\
\hline & Full & $11,225.0$ & & 88.82 & - & & 89.44 & - & \\
\hline Leuk2 & MDL+LFS & 4.3 & & 99.08 & - & & 90.00 & - & 231.15 \\
\hline Leukz & $\mathrm{MDL}+\mathrm{CON}$ & 3.0 & & 99.44 & - & & 85.56 & - & 17.54 \\
\hline & MDL+CFS & 79.0 & & 100.00 & $=$ & & 98.89 & + & 5407.43 \\
\hline & MChi2 & 166.1 & & 99.56 & - & & 93.33 & - & 904.96 \\
\hline & PPSO & 86.7 & 100.00 & 100.00 & & 100.00 & 96.74 & & 441.95 \\
\hline & Full & $10,367.0$ & & 63.52 & - & & 62.50 & - & \\
\hline Brain2 & MDL+LFS & 5.6 & & 98.80 & - & & 53.33 & - & 179.83 \\
\hline Díñ & MDL+CON & 4.7 & & 89.92 & - & & 61.67 & - & 19.33 \\
\hline & MDL+CFS & 63.4 & & 100.00 & $=$ & & 71.25 & + & 3304.74 \\
\hline & MChi2 & 160.7 & & 84.00 & - & & 70.00 & $=$ & 562.42 \\
\hline & PPSO & 66.7 & 100.00 & 99.99 & & 74.58 & 68.75 & & 357.34 \\
\hline & Full & $10,509.0$ & & 82.08 & - & & 85.33 & - & \\
\hline Prostate & MDL+LFS & 4.9 & & 82.44 & - & & 73.17 & - & 227.68 \\
\hline Fiostate & MDL+CON & 4.7 & & 98.46 & - & & 70.50 & - & 23.61 \\
\hline & MDL+CFS & 51.6 & & 98.12 & - & & 90.17 & - & 2694.27 \\
\hline & MChi2 & 33.6 & & 95.44 & - & & 86.17 & - & 1246.46 \\
\hline & PPSO & 65.6 & 100.00 & 99.84 & & 95.17 & 91.82 & & 478.17 \\
\hline & Full & $12,600.0$ & & 71.59 & - & & 78.05 & - & \\
\hline Lung & MDL+LFS & 12.2 & & 95.12 & - & & 80.55 & $=$ & 685.78 \\
\hline Lung & $\mathrm{MDL}+\mathrm{CON}$ & 6.6 & & 89.54 & - & & 74.64 & - & 63.37 \\
\hline & MDL+CFS & NA & & NA & & & NA & & NA \\
\hline & MChi2 & NA & & NA & & & NA & & NA \\
\hline & PPSO & 203.0 & 97.67 & 97.09 & & 84.11 & 79.38 & & 1335.21 \\
\hline & Full & $12,533.0$ & & 71.01 & - & & 71.42 & - & \\
\hline 11Tumor & MDL+LFS & 14.3 & & 79.96 & - & & 61.71 & - & 555.99 \\
\hline 11 1 1umor & MDL+CON & 9.4 & & 72.58 & - & & 53.83 & - & 85.41 \\
\hline & MDL+CFS & NA & & NA & & & NA & & NA \\
\hline & MChi2 & 2098.0 & & 93.32 & - & & 84.54 & + & 3125.14 \\
\hline & PPSO & 167.0 & 99.47 & 99.14 & & 83.20 & 76.83 & & 1387.01 \\
\hline
\end{tabular}




\section{E. PPSO versus Traditional Methods}

Table IV shows the compared results of PPSO with "Full", MDL+LFS, MDL+CON, MDL+CFS and MChi2. Besides the size and time shown in the third and the last columns, the best and the mean of the training and test accuracies are shown under each corresponding column. The " $S_{T r}$ " and " $S_{T e}$ " display the Wilcoxon significance test results of the corresponding method over PPSO in terms of training and test accuracy, respectively. The result of MChi2 on the Lung dataset is unavailable due to the out of memory error. Since CFS method is quite expensive, it fails to finish its run in 12 hours for the two largest datasets which are Lung and 11Tumor with more than 12,000 features.

As can be seen from Table IV that PPSO outperforms MDL+LFS and MDL+CON on all the ten datasets in terms of training accuracy and nine datasets in terms of test accuracy. Compared with MDL+LFS, PPSO achieves 6\% to $19 \%$ higher test accuracy on all datasets except for the Lung dataset where both obtain similar accuracy. However, the best test accuracy achieved by PPSO is $4 \%$ higher than MDL+LFS on this dataset. Similarly, PPSO achieves 5\% to $31 \%$ higher test accuracy than MDL+CON on all datasets except for DLBCL where MDL+CON achieves the highest average test result. Although these two methods obtain the smallest feature subsets, their test accuracies are usually the lowest among the compared methods. Compared with the Full accuracy, MDL+LFS even has $13 \%$ lower on Brain1 and MDL+CON has $18 \%$ lower on 11 Tumor. In contrast, PPSO achieves better accuracy than the full feature sets on all datasets.

Compared with MDL+CFS, PPSO obtains better or similar training accuracies on the eight datasets. For testing, PPSO achieves better results than MDL+CFS on three datasets namely 9Tumor, Leuk1 and Prostate. For the other five datasets, MDL+CFS has better results than PPSO on average but the best accuracy of PPSO is almost always better than MDL+CFS. It is noticed that the computation time of MDL+CFS quickly increases with the number of features. Therefore, scalability is a major concern when applying this method on high-dimensional data, such as Lung and 11Tumor.

Similarly, in terms of training accuracy, PPSO also outperforms MChi2 on all datasets. In terms of test accuracy, PPSO performs either similar to or significantly better than MChi2 on seven datasets with smaller feature subsets on four datasets. It is noticed that although MChi2 is a filter method, its running time is longer than that of PPSO and MDL+LFS on almost all datasets. While the difference in running time between MChi2 and PPSO is not too big on small datasets, it becomes much larger on datasets with more than ten thousand features. This indicates that the proposed algorithm is scalable to high-dimensional datasets.

\section{FURTHER ANALYSIS}

In addition to classification performance and feature subset size, generalisation and robustness are also important criteria in evaluating the performance of a FS method. While generalisability relates to the ability of the learned model to have a similar performance on both training and test data,robustness
TABLE V

AVERAGE TRAINING ACCURACY.

\begin{tabular}{|c|c|c|c|c|c|c|c|}
\hline Dataset & Method & Train-Acc(Std) & $T$ & Dataset & Method & Train-Acc(Std) & $T$ \\
\hline \multirow{3}{*}{ SRBCT } & PSO-FS & $100.00(0.00)$ & \multirow{3}{*}{$\begin{array}{l}= \\
=\end{array}$} & \multirow{3}{*}{ Leuk2 } & PSO-FS & $100.00(0.00)$ & \\
\hline & EPSO & $100.00(0.00)$ & & & EPSO & 100.00 & \\
\hline & PPSO & $100.00(0.00)$ & & & PPSO & $100.00(0.00)$ & \\
\hline \multirow{3}{*}{ DLBCL } & PSO-FS & 100.00( & \multirow{3}{*}{$=$} & \multirow{3}{*}{ Brain2 } & PSO-FS & $99.73(0.12)$ & \\
\hline & EPSO & 100.00( & & & EPSO & 98.38 & \\
\hline & PPSO & 100.00( & & & PPSO & 99.99 & \\
\hline \multirow{3}{*}{ 9Tumor } & PSO-FS & 97.49 & + & \multirow{3}{*}{ Prostate } & PSO-FS & 98.89 & \\
\hline & EPSO & 95.03 & \multirow[t]{2}{*}{+} & & EPSO & 98.56 & \\
\hline & PPSO & 92.31 & & & PPSO & 99.84 & \\
\hline \multirow{3}{*}{ Leuk1 } & PSO-FS & 100.00 & \multirow{3}{*}{$\begin{array}{l}= \\
=\end{array}$} & \multirow{3}{*}{ Lung } & PSO-FS & $97.77(0.05)$ & \\
\hline & EPSO & 100.00 & & & EPSO & $97.10(0.14)$ & \\
\hline & PPSO & 100.00 & & & PPSO & $97.09(0.30)$ & \\
\hline \multirow[t]{3}{*}{ Brain1 } & PSO-FS & $100.00(0.00)$ & \multirow{3}{*}{$\begin{array}{l}= \\
-\end{array}$} & \multirow{3}{*}{ 11Tumor } & PSO-FS & $99.80(0.08)$ & \\
\hline & & $\begin{array}{l}99.33 \\
99.99\end{array}$ & & & EPSC & (0.19) & \\
\hline & & 99.96 & & & $\mathrm{SO}$ & $99.14(0.20)$ & \\
\hline
\end{tabular}

relates to the ability to reproduce the results regardless of the small variance of the experiments. In this section, we compared the performance of the one-stage and two-stage approaches in terms of these two aspects. Furthermore, we also compared the performance of PPSO and the traditional ones in the FS bias scenario. By using the whole dataset for FS training, the performance difference between them reveals their learning capability.

\section{A. Generalisation and Robustness}

To compare the generalisability of the one-stage and twostage approaches, we examined the training accuracy of the returned solutions. Table V shows the mean and standard deviation of the training accuracy obtained by the three methods in 30 runs of each dataset.

As can be seen in Table V that PPSO achieves better training accuracy than PSO-FS on two datasets and similar on five. However, as seen in Table III, PPSO achieves significantly better test accuracy on seven datasets than PSO-FS and similar on one. This indicates that PPSO has better generalisability than PSO-FS.

Compared with EPSO, PPSO obtains the same training accuracy $(100 \%)$ on SRBCT with a smaller number of features and $3 \%$ higher accuracy on 11Tumor with a bigger feature subset. This indicates that the scaling mechanism helps PPSO effectively select appropriate feature subsets that can maintain or increase the training accuracy. However, these solutions seem to overfit the training data when they get $1 \%$ and $3 \%$ lower test accuracy than those of EPSO, respectively as shown in Table III. Another possible reason is that PPSO uses the fix potential cut-points calculated from the training data which may not be representative to the test data. A better mechanism to calculate the potential cut-points may help to improve the performance of PPSO.

We also note a big difference between the test and training accuracy (shown in Table III and V), especially in 9Tumor. This indicates the existence of overfitting which may be because the skew distribution of features in these datasets creates different distributions in training and test sets. Therefore, the learned model may not be generalised well to the test data. This problem is worse in datasets with smaller numbers of samples, such as Brain2 and 9Tumor with 50 and 60 instances. On top of this, the large number of classes as well 

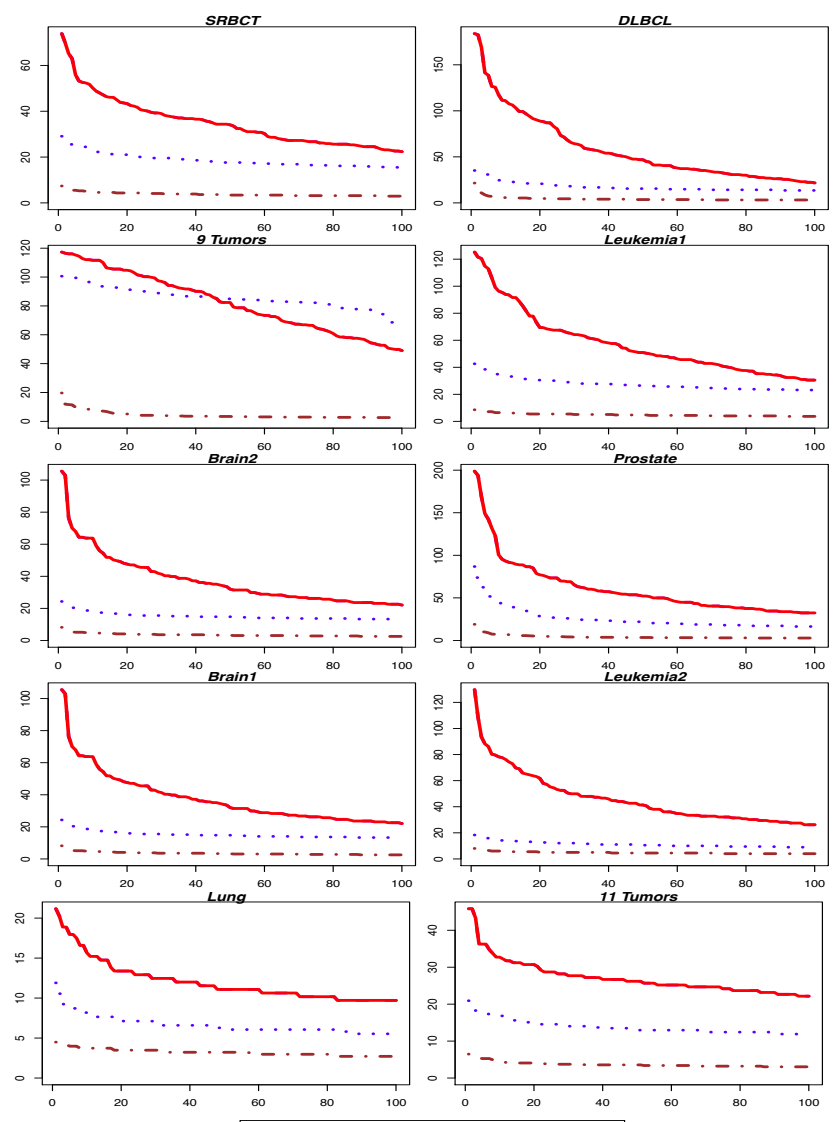

Fig. 7. Z-score of the top 100 selected features on each dataset.

as the class imbalance nature are additional challenges of these datasets. With 9 classes, 9Tumor has worse results than Brain2 which has 4 classes. As can be seen from Table IV that MDL+LFS and MChi2 performances are also affected by this phenomenon. The gaps between training and test accuracy obtained by MDL+LFS on 9Tumor and Brain2 are about $40 \%$ which is even larger than PPSO with about $30 \%$.

Since each method was run 30 times on each 10-fold $\mathrm{CV}$, it produced 300 solutions for a dataset. If the dataset has redundant features, different solutions may include very different features if the method is sensitive to the experimental conditions, leading to a low selection frequency of each feature. Therefore, we used the Z-score [56], [60] of 100 most selected features to examine the robustness of the compared methods. Z-score is a measure indicating the significance of the selection frequency of a feature. It is defined as follows:

$$
Z_{x}=\frac{f_{x}-\mu}{\sigma}
$$

where $f_{x}$ is the number of times feature $x$ being selected in the $L$ solutions ( $L$ is 300 in our experiment). $\mu$ and $\sigma$ are the mean and standard deviation of $f_{x}$. They are estimated based on the probability of feature $x$ being selected, $P\left(f_{x}\right)$, as follows:

$$
\mu=P\left(f_{x}\right) \cdot L
$$

and

$$
\sigma=\sqrt{P\left(f_{x}\right) \cdot\left(1-P\left(f_{x}\right)\right) \cdot L}
$$

where $P\left(f_{x}\right)$ is equal to the average number of selected features in $L$ solutions divided by the size of the original feature set. Therefore, the higher the value of $Z_{x}$, the less likely that feature $x$ is selected by chance. Therefore, an algorithm is said to be more robust if it selects more features with higher Z-scores.

Fig. 7 compares the Z-scores of the top 100 selected features by the three methods on each dataset. The chart shows that the Z-scores of the features selected by PPSO are greater than those of EPSO which are in turn greater than PSO-FS. This indicates that PPSO is more robust than the other methods.

In general, the results show that PPSO has better generalisability and robustness than PSO-FS. Solutions produced by PPSO are more compact and have higher discriminating power than PSO-FS. This demonstrates our hypothesis that important information including feature interaction may be lost during the discretisation process in the first stage of PSO-FS. Since the two-stage approach evaluates the selected features and their cut-points simultaneously, it takes these important information into account.

\section{B. Learning Capability}

To confirm the performance of the proposed approach, we conducted another experiment with $F S$ bias. In this experiment, the whole dataset is used to train the algorithm. Based on the evolved cut-points and the selected features, the whole dataset is transformed and used for performance evaluation. Besides KNN, Naive Bayes (NB) is also used to see if the evolved solutions by PPSO can also improve the performance of classifiers other than the one used in the fitness function.

Table VI shows the average of 10-fold CV classification accuracies obtained by $\mathrm{KNN}(\mathrm{K}=1)$ and $\mathrm{NB}$ on the original datasets ("Full") and the transformed datasets by five methods. Wilcoxon significance test with significance level of 0.05 was used to compare the classification accuracy obtained by $\mathrm{KNN}$ and $\mathrm{NB}$ between the corresponding method and PPSO. " $S_{K}$ " and " $S_{N B}$ " present the statistical test results of the corresponding method over PPSO using KNN and NB, respectively.

According to Table VI, PPSO obtains 100\% accuracy in all or almost all 30 runs on seven datasets for KNN and six datasets for NB. For KNN, its results outperform MDL+LFS, MDL+CON and MChi 2 on 8, 9, and 7 datasets, respectively. It is noticed that MDL+LFS performs quite well on datasets with a small number of classes; however, its accuracy is $20 \%$ lower than that of PPSO on 9Tumor and 11Tumor datasets. MDL+CON also suffers from this problem with an even a bigger gap. Compared with MDL+CFS, PPSO obtains a significantly better results on 2 datasets, similar in 6 and worse in 2. It is also noticed that even though MDL+CFS achieves comparable performance with PPSO, its running time is 4 to 100 times longer than that of PPSO. In general, with totally 49 comparisons (excluding the unavailable result of MChi2 on Lung dataset), PPSO wins 36 cases, draws 10 and loses 3. 
TABLE VI

RESULTS WITH FEATURE SELECTION BIAS.

\begin{tabular}{|c|c|c|c|c|c|c|c|c|c|}
\hline \multirow{2}{*}{ Dataset } & \multirow{2}{*}{ Method } & \multirow{2}{*}{ Size } & \multirow{2}{*}{ Time (s) } & \multicolumn{3}{|c|}{ KNN } & \multicolumn{3}{|c|}{ Naive Bayes } \\
\hline & & & & Best & Mean & $S_{K}$ & Best & Mean & $S_{N B}$ \\
\hline \multirow{6}{*}{ SRBCT } & Full & $2,308.0$ & & & 83.35 & - & \multirow[b]{6}{*}{100.00} & 99.17 & - \\
\hline & MDL+LFS & 8.0 & 5.52 & & 99.14 & - & & 99.14 & - \\
\hline & $\mathrm{MDL}+\mathrm{CON}$ & 4.0 & 0.53 & & 95.18 & - & & 95.18 & - \\
\hline & $\mathrm{MDL}+\mathrm{CFS}$ & 80.0 & 36.53 & & 100.00 & $=$ & & 100.00 & $=$ \\
\hline & MChi2 & 120.0 & 9.80 & & 100.00 & $=$ & & 100.00 & $=$ \\
\hline & PPSO & 114.4 & 9.51 & 100.00 & 99.97 & & & 100.00 & \\
\hline \multirow{6}{*}{ DLBCL } & Full & $5,469.0$ & & & 81.71 & - & \multirow[b]{6}{*}{100.00} & \begin{tabular}{|l|}
77.17 \\
\end{tabular} & - \\
\hline & MDL+LFS & 9.0 & 11.09 & & 97.37 & - & & 97.41 & - \\
\hline & $\mathrm{MDL}+\mathrm{CON}$ & 4.0 & 0.64 & & 96.10 & - & & 96.10 & - \\
\hline & $\mathrm{MDL}+\mathrm{CFS}$ & 55.0 & 132.31 & & 100.00 & $=$ & & 100.00 & $=$ \\
\hline & MChi2 & 15.0 & 37.80 & & 87.79 & - & & 90.43 & - \\
\hline & PPSO & 49.7 & 21.86 & 100.00 & 100.00 & & & 100.00 & \\
\hline \multirow{6}{*}{ 9Tumor } & Full & $5,726.0$ & & & 33.44 & $\begin{array}{lll}- & & \end{array}$ & \multirow[b]{6}{*}{81.44} & 40.00 & - \\
\hline & MDL+LFS & 13.0 & 6.09 & & 74.49 & - & & 65.70 & - \\
\hline & $\mathrm{MDL}+\mathrm{CON}$ & 6.0 & 0.88 & & 68.33 & - & & 68.33 & - \\
\hline & $\mathrm{MDL}+\mathrm{CFS}$ & 44.0 & 77.68 & & 89.97 & - & & 94.60 & + \\
\hline & MChi2 & 78.0 & 26.72 & & 77.60 & - & & 66.14 & - \\
\hline & PPSO & 113.5 & 23.61 & 98.77 & 95.83 & & & 78.09 & \\
\hline \multirow{6}{*}{ Leuk1 } & Full & $5,327.0$ & & & 79.77 & - & & 90.00 & - \\
\hline & MDL+LFS & 7.0 & 7.40 & & 96.30 & - & & 100.00 & $=$ \\
\hline & $\mathrm{MDL}+\mathrm{CON}$ & 4.0 & 0.63 & & 98.61 & - & & 98.61 & - \\
\hline & $\mathrm{MDL}+\mathrm{CFS}$ & 74.0 & 221.60 & & 100.00 & $=$ & & 100.00 & $=$ \\
\hline & MChi2 & 66.0 & 372.90 & & 99.12 & - & & 99.12 & - \\
\hline & PPSO & 90.3 & 25.10 & 100.00 & 100.00 & & 100.00 & 100.00 & \\
\hline & Full & $5,920.0$ & & & 65.07 & - & & 77.50 & - \\
\hline Brain1 & MDL+LFS & 10.0 & 12.10 & & 84.33 & - & & 76.33 & - \\
\hline & $\mathrm{MDL}+\mathrm{CON}$ & 5.0 & 0.94 & & 93.33 & - & & 93.33 & - \\
\hline & $\mathrm{MDL}+\mathrm{CFS}$ & 103.0 & 385.85 & & 100.00 & $=$ & & 100.00 & $=$ \\
\hline & MChi2 & 325.0 & 130.96 & & 81.00 & - & & 83.00 & - \\
\hline & PPSO & 83.9 & 31.87 & 100.00 & 100.00 & & 100.00 & 99.88 & \\
\hline & Full & $11,225.0$ & & & 88.82 & - & & 95.00 & - \\
\hline Leuk2 & MDL+LFS & 6.0 & 32.10 & & 98.61 & - & & 100.00 & $=$ \\
\hline & $\mathrm{MDL}+\mathrm{CON}$ & 3.0 & 1.39 & & 98.61 & - & & 98.61 & - \\
\hline & $\mathrm{MDL}+\mathrm{CFS}$ & 87.0 & 1079.57 & & 100.00 & $=$ & & 100.00 & $=$ \\
\hline & MChi2 & 300.0 & 46.86 & & 100.00 & $=$ & & 100.00 & $=$ \\
\hline & PPSO & 95.6 & 44.72 & 100.00 & 99.92 & & 100.00 & 99.84 & \\
\hline & Full & $10,367.0$ & & & 63.52 & - & & 66.67 & - \\
\hline Brain2 & MDL+LFS & 6.0 & 26.36 & & 100.00 & $=$ & & 100.00 & $=$ \\
\hline & $\mathrm{MDL}+\mathrm{CON}$ & 3.0 & 1.24 & & 94.00 & - & & 94.00 & - \\
\hline & $\mathrm{MDL}+\mathrm{CFS}$ & 76.0 & 756.38 & & 100.00 & $=$ & & 100.00 & $=$ \\
\hline & MChi2 & 110.0 & 64.52 & & 94.64 & - & & 92.86 & - \\
\hline & PPSO & 82.1 & 36.63 & 100.00 & 100.00 & & 100.00 & 99.94 & \\
\hline & Full & $10,509.0$ & & & 82.08 & - & & 62.17 & - \\
\hline Prostate & MDL+LFS & 12.0 & 20.80 & & 96.04 & - & & 98.04 & + \\
\hline & $\mathrm{MDL}+\mathrm{CON}$ & 4.0 & 1.78 & & 100.00 & + & & 100.00 & $=$ \\
\hline & $\mathrm{MDL}+\mathrm{CFS}$ & 55.0 & 532.78 & & 98.00 & - & & 99.04 & + \\
\hline & MChi2 & 50.0 & 30.90 & & 94.08 & - & & 95.15 & - \\
\hline & PPSO & 70.8 & 51.58 & 100.00 & 99.30 & & 99.04 & 96.63 & \\
\hline & Full & $12,600.0$ & & & 71.59 & - & & 79.98 & - \\
\hline Lung & MDL+LFS & 12.0 & 87.10 & & 96.77 & $=$ & & 95.10 & + \\
\hline & $\mathrm{MDL}+\mathrm{CON}$ & 5.0 & 5.40 & & 93.60 & - & & 93.60 & + \\
\hline & $\mathrm{MDL}+\mathrm{CFS}$ & 286.0 & 17568.40 & & 98.76 & + & & 99.86 & + \\
\hline & MChi2 & NA & NA & & NA & & & NA & \\
\hline & PPSO & 218.4 & 175.81 & 97.90 & 96.65 & & 93.13 & 84.88 & \\
\hline & Full & $12,533.0$ & & & 71.01 & - & & 77.47 & - \\
\hline 11Tumor & MDL+LFS & 12.0 & 41.95 & & 78.58 & - & & 78.18 & - \\
\hline & $\mathrm{MDL}+\mathrm{CON}$ & 7.0 & 6.57 & & 72.99 & - & & 72.99 & - \\
\hline & $\mathrm{MDL}+\mathrm{CFS}$ & 207.0 & 7911.05 & & 98.89 & + & & 100.00 & + \\
\hline & MChi2 & 2643.0 & 100.07 & & 94.16 & - & & 96.29 & $=$ \\
\hline & PPSO & 173.5 & 162.15 & 99.66 & 98.44 & & 98.23 & 96.10 & \\
\hline
\end{tabular}

Similarly, the transformed datasets by PPSO also help NB improve its classification accuracy on all datasets, six of which reach $100 \%$ accuracy in all or almost all 30 runs. Compared with feature subsets obtained by MDL+LFS, MDL+CON and MChi2, those of PPSO achieve either similar or better classification performance on eight to nine datasets. Compared with MDL+CFS, PPSO obtains similar performance on six datasets and worse in the remaining four. On Brain1, while MDL+LFS obtains $76.33 \%$ with 10 features and MChi2 obtains $83 \%$ with 325 features, PPSO only selects 84 features to improve the NB accuracy to $99.88 \%$. Using over ten times longer time than PPSO, MDL+CFS obtains $100 \%$ accuracy on this dataset however with 105 features. In general, for NB, PPSO wins 29 cases, draws 13 and loses 7 in the total 49 comparisons.

It is also noticed that the result subsets of PPSO and MDL+CFS on 9Tumor dataset have opposite effects on KNN and NB. While the transformed dataset of PPSO performs better than that of MDL+CFS in KNN, it obtains worse result than MDL+CFS in NB. Given that PPSO selects many more features than MDL+CFS in this dataset, this phenomenon might have happened because the feature subset evolved by PPSO still contains redundant or correlated features which may not strongly affect the performance of KNN but may negatively affect the performance of NB. Since PPSO uses $\mathrm{KNN}$ as a learning algorithm to evaluate the feature subset, the redundant features are not well identified. On the other hand, CFS is good at recognising correlated or redundant features. This possible reason also explains the loses of PPSO on the remaining three datasets namely Prostate, Lung and 11Tumor.

\section{CONCLUSIONS AND Future WorK}

The goal of this study was to propose an integrating approach to discretisation and FS in a single stage using BBPSO. The goal was achieved by proposing a new PSO-based methods, PPSO, with a new PSO representation to choose cutpoints for discretising multiple features and selecting features simultaneously. PPSO was compared with using the full set of original features, EPSO, and the two-stage approach (PSOFS).

Experimental results on ten datasets having thousands to tens of thousands of features with variant numbers of classes show that PPSO can discretise multiple features simultaneously and select a much smaller number of relevant features with better discriminating ability. Comparison between PPSO and PSO-FS suggests that it is more effective to combine discretisation and FS in a single stage. Compared with EPSO, PPSO obtains either equivalent or better results with smaller numbers of features. Further analysis also shows that PPSO is more general and more robust than the compared PSO methods.

PPSO was also compared with four traditional methods representing for two-stage and one-stage approaches, MDL+LFS, MDL+CON, MDL+CFS and MChi2. The results of two experiments with and without FS bias show that PPSO has significantly better performance than MDL+LFS, MDL+CON and MChi2 and similar performance to MDL+CFS in most cases. The results also show that PPSO is more scalable than MDL+CFS and MChi2 in dealing with high-dimensional problems. Compared results on both $\mathrm{KNN}$ and $\mathrm{NB}$ indicate that solutions obtained by PPSO can be generalised to other classifier than the one used during the training process.

As a binary discretisation method, PPSO may not work well on data that needs to be discretised into multiple intervals. Furthermore, the potential cut-points are calculated based on MDLP. Therefore, investigation of using other schemes for cut-point calculation, multiple interval discretisation, and different approaches to optimising the search process will be included in our future work.

Empirical results show that the running time of PPSO is scalable to high-dimensional data with $10,000+$ features. 
However, since the PPSO representation is static and proportional to the feature set size, applying PPSO to datasets with $100,000+$ features may be limited by the memory capacity. Furthermore, the search space will become much larger due to the exponential increase of the possible solutions, so it may require a different way to solve the problem. In the future, we will investigate using a dynamic representation in PPSO to overcome this limitation.

\section{ACKNOWLEDGMENT}

This work was supported in part by the Marsden Fund of New Zealand Government under Contract VUW1209, VUW1509 and 16-VUW-111, Huawei Industry Fund E2880/3663, and the University Research Fund at Victoria University of Wellington 209861/3580, 209862/3580, and $213150 / 3662$

\section{REFERENCES}

[1] A. J. Ferreira and M. A. Figueiredo, "Efficient feature selection filter for high-dimensional data," Pattern Recognition Letters, vol. 33, no. 13, pp. 1794-1804, 2012.

2] B. Tran, B. Xue, and M. Zhang, "Improved PSO for Feature Selection on High-Dimensional Datasets," in Simulated Evolution and Learning, ser. Lecture Notes in Computer Science, 2014, vol. 8886, pp. 503-515.

[3] C. Ding and H. Peng, "Minimum redundancy feature selection from microarray gene expression data," Journal of bioinformatics and computational biology, vol. 3, no. 02, pp. 185-205, 2005.

[4] J. Dougherty, R. Kohavi, M. Sahami et al., "Supervised and unsupervised discretization of continuous features," in Machine learning: proceedings of the twelfth international conference, vol. 12, 1995, pp. 194-202.

[5] V. Bolón-Canedo, N. Sánchez-Maroño, and A. Alonso-Betanzos, "On the effectiveness of discretization on gene selection of microarray data," in The International Joint Conference on Neural networks (IJCNN), IEEE, 2010, pp. 1-8.

[6] A. J. Ferreira and M. A. Figueiredo, "An unsupervised approach to feature discretization and selection," Pattern Recognition, vol. 45, no. 9, pp. 3048-3060, 2012.

[7] S. Chao and Y. Li, "Multivariate interdependent discretization for continuous attribute," in Third International Conference on Information Technology and Applications, vol. 1. IEEE, 2005, pp. 167-172.

[8] R. Eberhart and J. Kennedy, "A new optimizer using particle swarm theory," in Proceedings of the Sixth International Symposium on Micro Machine and Human Science, 1995, pp. 39-43.

[9] B. Xue, M. Zhang, and W. Browne, "Particle swarm optimization for feature selection in classification: A multi-objective approach," IEEE Transactions on Cybernetics, vol. 43, no. 6, pp. 1656-1671, 2013.

[10] B. Xue, M. Zhang, and W. N. Browne, "Particle swarm optimisation for feature selection in classification: Novel initialisation and updating mechanisms," Applied Soft Computing, vol. 18, no. 0, pp. 261-276, 2014.

[11] L. Cervante, B. Xue, M. Zhang, and L. Shang, "Binary particle swarm optimisation for feature selection: A filter based approach," in IEEE Congress on Evolutionary Computation (CEC'12), 2012, pp. 881-888.

[12] M. Mohamad, S. Omatu, S. Deris, and M. Yoshioka, "A modified binary particle swarm optimization for selecting the small subset of informative genes from gene expression data," Information Technology in Biomedicine, vol. 15, no. 6, pp. 813-822, 2011.

[13] W. Zhou and J. A. Dickerson, "A novel class dependent feature selection method for cancer biomarker discovery," Computers in biology and medicine, vol. 47, pp. 66-75, 2014

[14] B. Tran, B. Xue, and M. Zhang, "Bare-bone particle swarm optimisation for simultaneously discretising and selecting features for highdimensional classification," in European Conference on Applications of Evolutionary Computation (EvoApplications). Springer, 2016, pp. 701718.

[15] J. Kennedy, "Bare bones particle swarms," in Proceedings of the IEEE Swarm Intelligence Symposium (SIS)., 2003, pp. 80-87.

[16] U. M. Fayyad and K. B. Irani, "Multi-interval discretization of continuous-valued attributes for classification learning," in Thirteenth International Joint Conference on Articial Intelligence, vol. 2. Morgan Kaufmann Publishers, 1993, pp. 1022-1027.
[17] S. K. Singhi and H. Liu, "Feature subset selection bias for classification learning," in Proceedings of the 23rd international conference on Machine learning. ACM, 2006, pp. 849-856.

[18] R. Kohavi and G. H. John, "Wrappers for feature subset selection," Artificial Intelligence, vol. 97, no. 1-2, pp. 273-324, 1997.

[19] J. Liang, S. Yang, and A. Winstanley, "Invariant optimal feature selection: A distance discriminant and feature ranking based solution," Pattern Recognition, vol. 41, no. 5, pp. 1429-1439, 2008.

[20] B. Xue, L. Cervante, L. Shang, W. Browne, and M. Zhang, "A multiobjective particle swarm optimisation for filter-based feature selection in classification problems," Connection Science, vol. 24, no. 2-3, pp. 91-116, 2012.

[21] M. Dash and H. Liu, "Consistency-based search in feature selection," Artificial Intelligence, vol. 151, pp. 155-176, 2003.

[22] M. A. Hall, "Correlation-based feature selection for discrete and numeric class machine learning," in Proceedings of the Seventeenth International Conference on Machine Learning, 2000, pp. 359-366.

[23] T. Cover and P. Hart, "Nearest neighbor pattern classification," IEEE Transactions on Information Theory, vol. 13, no. 1, pp. 21-27, 1967.

[24] T. Mitchell, Machine Learning. McGraw-Hill, 1997.

[25] C.-W. Hsu, C.-C. Chang, and C.-J. Lin, "A practical guide to support vector classification," Tech. Rep., 2003.

[26] T. Butler-Yeoman, B. Xue, and M. Zhang, "Particle swarm optimisation for feature selection: A hybrid filter-wrapper approach," in IEEE Congress on Evolutionary Computation, 2015, pp. 2428-2435.

[27] M. Dash and H. liu, "Feature selection for classification," Intelligent data analysis, vol. 1, no. 4, pp. 131-156, 1997.

[28] M. Gutlein, E. Frank, M. Hall, and A. Karwath, "Large-scale attribute selection using wrappers," in IEEE Symposium on Computational Intelligence and Data Mining, 2009, pp. 332-339.

[29] M. Hall, E. Frank, G. Holmes, B. Pfahringer, P. Reutemann, and I. H. Witten, "The weka data mining software: an update," ACM SIGKDD explorations newsletter, vol. 11, no. 1, pp. 10-18, 2009.

[30] A. Whitney, "A direct method of nonparametric measurement selection," IEEE Transactions on Computers, vol. C-20, no. 9, pp. 1100-1103, 1971.

[31] T. Marill and D. M. Green, "On the effectiveness of receptors in recognition systems." IEEE Transactions on Information Theory, vol. 9, no. 1, pp. 11-17, 1963.

[32] I. Guyon and A. Elisseeff, "An introduction to variable and feature selection," Journal of Machine Learning Research, vol. 3, pp. 11571182, 2003.

[33] H. B. Nguyen, B. Xue, I. Liu, and M. Zhang, "Filter based backward elimination in wrapper based pso for feature selection in classification," in IEEE Congress on Evolutionary Computation, 2014, pp. 3111-3118.

[34] H. Liu and R. Setiono, "Feature selection and classification-a probabilistic wrapper approach," in Proceedings of 9th International Conference on Industrial and Engineering Applications of AI and ES, 1997, pp. 419-424.

[35] A. Unler and R. B. C. Alper Murat, "mr2pso: A maximum relevance minimum redundancy feature selection method based on swarm intelligence for support vector machine classification," Information Sciences, vol. 20 , pp. 4625-4641, 2011.

[36] B. Xue, M. Zhang, W. N. Browne, and X. Yao, "A survey on evolutionary computation approaches to feature selection," IEEE Transactions on Evolutionary Computation, vol. 20, no. 4, pp. 606-626, 2016.

[37] B. Tran, B. Xue, and M. Zhang, "Overview of Particle Swarm Optimisation for Feature Selection in Classification," in Simulated Evolution and Learning, ser. Lecture Notes in Computer Science. Springer, 2014 vol. 8886, pp. 605-617.

[38] S. Garcia, J. Luengo, J. A. Sáez, V. Lopez, and F. Herrera, "A survey of discretization techniques: taxonomy and empirical analysis in supervised learning," Knowledge and Data Engineering, IEEE Transactions on, vol. 25, no. 4, pp. 734-750, 2013.

[39] H. Liu, F. Hussain, C. L. Tan, and M. Dash, "Discretization: An enabling technique," Data mining and knowledge discovery, vol. 6, no. 4, pp. 393-423, 2002.

[40] S. Kotsiantis and D. Kanellopoulos, "Discretization techniques: A recent survey," GESTS International Transactions on Computer Science and Engineering, vol. 32, no. 1, pp. 47-58, 2006.

[41] J. Catlett, "On changing continuous attributes into ordered discrete attributes," in Machine learning-EWSL-91. Springer, 1991, pp. 164178.

[42] R. C. Holte, "Very simple classification rules perform well on most commonly used datasets," Machine learning, vol. 11, no. 1, pp. 63-90, 1993. 
[43] J. W. Grzymala-Busse, "Discretization based on entropy and multiple scanning," Entropy, vol. 15, no. 5, pp. 1486-1502, 2013.

[44] A. Cano, D. T. Nguyen, S. Ventura, and K. J. Cios, "ur-CAIM: improved CAIM discretization for unbalanced and balanced data," Soft Computing, pp. 1-16, 2014.

[45] P. Yang, J.-S. Li, and Y.-X. Huang, "HDD: a hypercube division-based algorithm for discretisation," International Journal of Systems Science, vol. 42 , no. 4 , pp. 557-566, 2011.

[46] J. L. Flores, I. Inza, and P. Larrañaga, "Wrapper discretization by means of estimation of distribution algorithms," Intelligent Data Analysis, vol. 11 , no. 5, pp. 525-545, 2007.

[47] S. Ramirez-Gallego, S. Garcia, J. Benitez, and F. Herrera, "Multivariate discretization based on evolutionary cut points selection for classification," IEEE Transactions on Cybernetics, vol. 46, no. 3, pp. 595-608, March 2016.

[48] P. Mahanta, H. A. Ahmed, J. K. Kalita, and D. K. Bhattacharyya, "Discretization in gene expression data analysis: a selected survey," in Proceedings of the Second International Conference on Computational Science, Engineering and Information Technology. ACM, 2012, pp. 69-75.

[49] H. Liu and R. Setiono, "Chi2: Feature selection and discretization of numeric attributes," in tai. IEEE, 1995, p. 388.

[50] F. E. Tay and L. Shen, "A modified chi2 algorithm for discretization," IEEE Transactions on Knowledge and Data Engineering, vol. 14, no. 3 , pp. 666-670, 2002.

[51] L. Jaba Sheela and D. V. Shanthi, "An approach for discretization and feature selection of continuous-valued attributes in medical images for classification learning," International Journal of Computer Theory and Engineering, vol. 1, no. 2, pp. 154-158, 2009.

[52] A. Ferreira and M. Figueiredo, "Unsupervised joint feature discretization and selection," in Pattern Recognition and Image Analysis. Springer, 2011, pp. 200-207.

[53] F. Van den Bergh and A. P. Engelbrecht, "A study of particle swarm optimization particle trajectories," Information sciences, vol. 176, no. 8 , pp. 937-971, 2006.

[54] G. Chandrashekar and F. Sahin, "A survey on feature selection methods," Computers \& Electrical Engineering, vol. 40, pp. 16-28, 2014.

[55] Y. Zhang, D. Gong, Y. Hu, and W. Zhang, "Feature selection algorithm based on bare bones particle swarm optimization," Neurocomputing, vol. 148, pp. $150-157,2015$.

[56] Z. Zhu, Y.-S. Ong, and M. Dash, "Markov blanket-embedded genetic algorithm for gene selection," Pattern Recognition, vol. 40, no. 11, pp. 3236-3248, 2007.

[57] G. Patterson and M. Zhang, "Fitness functions in genetic programming for classification with unbalanced data," in Advances in Artificial Intelligence. Springer, 2007, pp. 769-775.

[58] H. Al-Sahaf, M. Zhang, M. Johnston, and B. Verma, "Image descriptor: A genetic programming approach to multiclass texture classification," in IEEE Congress on Evolutionary Computation, 2015, pp. 2460-2467.

[59] J. Alcalá, A. Fernández, J. Luengo, J. Derrac, S. García, L. Sánchez, and F. Herrera, "Keel data-mining software tool: Data set repository, integration of algorithms and experimental analysis framework," Journal of Multiple-Valued Logic and Soft Computing, vol. 17, no. 255-287, p. $11,2010$.

[60] T. Jirapech-Umpai and S. Aitken, "Feature selection and classification for microarray data analysis: Evolutionary methods for identifying predictive genes," BMC bioinformatics, vol. 6, no. 1, p. 148, 2005

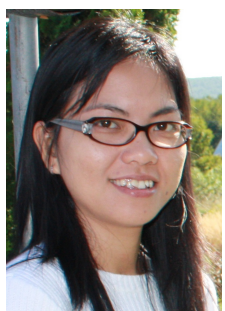

Binh Tran (S'14) received her B.E. in Computer Science from Cantho University, Vietnam, in 1998. She received her Master degree in Applied Computer Science from Free University of Brussels, Belgium, in 2002. Since 2014, she has joined the Evolutionary Computation Research Group (ECRG) at Victoria University of Wellington (VUW). Currently, she is a Ph.D. Candidate in the School of Engineering and Computer Science at VUW.

Binh's research interests are in evolutionary computation, feature manipulation including feature selection and construction, high dimensional data, and machine learning.

Ms. Tran is a member of the IEEE Computational Intelligence Society (CIS). She has been serving as a reviewer for international journals and conferences in the field.

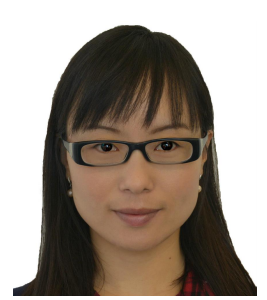

Bing Xue (M'10) received the B.Sc. degree from the Henan University of Economics and Law, Zhengzhou, China, in 2007, the M.Sc. degree in management from Shenzhen University, Shenzhen, China, in 2010, and the $\mathrm{PhD}$ degree in computer science in 2014 at Victoria University of Wellington, New Zealand.

She is currently a Senior Lecturer in School of Engineering and Computer Science at Victoria University of Wellington. Her research focuses mainly on evolutionary computation, feature selection, feature construction, multi-objective optimisation, data mining and machine learning. She has over 100 papers published in fully referred international journals and conferences.

Dr Xue is an Associate Editor/member of Editorial Board for five international journals including IEEE Computational Intelligence Magazine, Applied Soft Computing, International Journal of Swarm Intelligence, and International Journal of Computer Information Systems and Industrial Management Applications. She is a Guest Editor for the Special Issue on Evolutionary Feature Reduction and Machine Learning for the Springer Journal of Sof Computing. She is also a Guest Editor for Evolutionary Image Analysis and Pattern Recognition in Journal of Applied Soft Computing. She is serving as a reviewer of over 20 international journals including IEEE Transactions on Cybernetics and IEEE Transactions on Evolutionary Computation.

She has been a chair for a number of international conferences including the Leading Chair of IEEE Symposium on Computational Intelligence in Feature Analysis, Selection, and Learning in Image and Pattern Recognition at SSCI 2016 and 2017, a Program Co-Chair of the 31th Australasian AI 2018, ACALCI 2018, and the 7th SoCPaR 2015, Special Session Chair for ES2016, a Tutorial Chair for the 30th Australasian AI, publicity chair for SEAL 2017, and ICDIS 2018. She is the organiser of the special session on Evolutionary Feature Selection and Construction in IEEE Congress on Evolutionary Computation (CEC) 2015, 2016 and 2017, and SEAL 2014 and 2017.

Dr Xue is currently the Chair of the IEEE Task Force on Evolutionary Feature Selection and Construction. She is chairing the IEEE CIS Graduate Student Research Grants Committee.

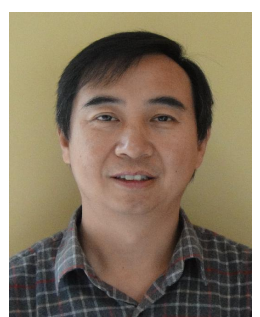

Mengjie Zhang (M'04-SM'10) received the B.E and M.E. degrees from Artificial Intelligence Research Center, Agricultural University of Hebei, Hebei, China, and the Ph.D. degree in computer science from RMIT University, Melbourne, VIC, Australia, in 1989, 1992, and 2000, respectively.

Since 2000, he has been with the Victoria University of Wellington, Wellington, New Zealand, where he is currently Professor of Computer Science, Head of the Evolutionary Computation Research Group and the Associate Dean (Research and Innovation) in the Faculty of Engineering. His current research interests include evolutionary computation, particularly genetic programming, particle swarm optimization, and learning classifier systems with application areas of image analysis, multiobjective optimization, feature selection and reduction, job shop scheduling, and transfer learning. He has published over 350 research papers in refereed international journals and conferences.

Prof. Zhang has been serving as an Associated Editor or Editorial Board Member for ten international journals (including IEEE Transactions on Evolutionary Computation, IEEE Transactions on Cybernetics, Evolutionary Computation Journal, and IEEE Transactions on Emergent Topics in CI) and as a Reviewer of over 20 international journals. He has been serving as a Steering Committee Member and a Program Committee Member for over 100 international conferences. He has supervised over 50 postgraduate research students. He is the Chair of the IEEE CIS Intelligent Systems and Applications Technical Committee, a member of the IEEE CIS Evolutionary Computation Technical Committee, a Vice-Chair of the IEEE CIS Task Force on Evolutionary Computer Vision and Image Processing, a Vice-Chair of the IEEE CIS Task Force on Evolutionary Computation for Feature Selection and Construction, a member of IEEE CIS Task Force of Hyper-heuristics, and the Founding Chair for IEEE Computational Intelligence Chapter in New Zealand. 